\title{
Die Fußnote, das unbekannte Wesen - Potential und Grenzen juristischer Zitationsanalyse
}

\author{
Hanjo Hamann*
}

A. Zitationsanalyse: Jurisprudentias verlorene Tochter.... 502

B. Cui bono: Jahrmarkt der Eitelkeiten oder wissenschaftliche Erkenntnisquelle?

C. Motivbündel: Eine kommunikationspsychologische Theorie der Fußnote. . 509

D. Praktische Erprobung: Pilotstudien zur Zitationsanalyse 512

I. Datenerhebung ..................... 512

II. Datenaufbereitung .................. 516

III. Datenauswertung ................. 518

1. Publikationsflut und dunkle Materie 518
2. Zeitschriftenranking 521

3. Einflussmessung einzelner Artikel.......................... 524

4. Klassiker der Aufsatzliteratur . . 527

IV. Diskussion ........................ 529

1. Geringe Stichprobengröße...... 529

2. Mangelnde Datenqualität und technische Fehler.............. 530

3. Beschränkung auf ein einziges Medium ........................ 530

E. Ausblick: Die Zukunft der Zitationsanalyse 532

Die juristische Fußnotenindustrie hat ein Image-Problem. Sie gilt als „Krebsleiden“ der Rechtswissenschaft, ${ }^{1}$ und die sprichwörtlichen „Zitatenfriedhöfe“ sind quer durch alle Rechtsgebiete berüchtigt. ${ }^{2}$ Doch beide Metaphern sind schief: Jene vom unauffällig schleichenden Tumor ebenso wie die vom stillen und besinnlichen Friedhof. Denn in Fußnoten wird der Diskurs nicht friedlich beerdigt, sondern kämpfend vollzogen: Sie sind die Arena eines subtilen Ringens um wissenschaftliche Vormacht, zugleich Brutstätte „herrschender“ und Richtplatz für „andere“ Meinungen:

„Was ist Sinn und Zweck der Fußnote? [...] eine Frage, die jeden Studienanfänger quält, wenn er zum ersten Mal in jene Unterwelt von Kurztexten eintaucht, aus der jeder wissenschaftliche Großtext wie durch ein Kanalisationssystem zugleich mit Belegen versorgt und von den abweichenden Lehrmeinungen unfähiger Kollegen entsorgt wird. Fußnoten sind beides: Nahrungszufubr und Verdauung, Bankett und Toilette, Gastmabl und Vomitorium (Ort zum Brechen). So wie ein modernes Haus erst durch Strom-und Wasserversorgung, Kanalisation und Müllabfubr zu einem zivilisierten Habitat wird, wird ein Text erst durch die Fußnoten wissenschaftlich. "3

* Dr. iur. Hanjo Hamann, Gastforscher am Max-Planck-Institut zur Erforschung von Gemeinschaftsgütern in Bonn und Nachwuchskollegiat der Heidelberger Akademie der Wissenschaften mit dem Projekt „Konzeption und Erschließung eines juristischen Referenzkorpus (JuReKo)“, gem. mit F. Vogel. Ich danke Prof. Ch. Engel, Dr. O. Himmler und besonders Y. Hermstrüwer für wertvolle Anregungen und Impulse.

1 K. F. Röhl/H. C. Röhl, Allgemeine Rechtslehre, 3. Aufl., Köln 2008, S. V Fn. *.

2 Vgl. die Buchbesprechungen von M. Heidenhain, NJW 1998, S. 2339; A. Junker, RdA 2010, S. 63; R. Müller-Metz, NZV 1996, S. 442 (443); W. Treiber, NVwZ 2000, S. 1275; D. Leuering, NZG 2005, S. 343 (344).

3 D. Schwanitz, Bildung. Alles was man wissen muss, 26. Aufl., Frankfurt am Main 2006, S. 360 Fn. $1=$ ders., Die Welt v. 24.1.1998 (online unter http://www.welt.de/print-welt/article598108/Wie-Streithaehne-die-zum-Kampf-mal-kurz-auf-die-Strasse-gehen.html). 
Auch für den Rechtsdiskurs haben Fußnoten „schlechthin konstituierende Bedeutung “, ${ }^{4}$ sie gelten gar als „Proprium der Wissenschaft" ${ }^{5}$ - und fristen dennoch „ein Schattendasein in der juristischen Fachliteratur “. ${ }^{6}$ Denn für den erlauchten Wissenschaftsbetrieb sind sie bloße Arbeitsmittel, denen ebenso wenig Aufmerksamkeit zukommt wie anderen Arbeitsmitteln, etwa dem Textmarker oder dem Notizzettel. Zum Gegenstand ernsthafter Untersuchungen werden Fußnoten daher selten. ${ }^{7}$

Anders als Textmarker und Notizzettel sind Fußnoten aber auch - und sogar in erster Linie - Kommunikationsmedien. ${ }^{8}$ Das erklärt und rechtfertigt ein wissenschaftliches Interesse an ihrer Untersuchung, das sich mit der Zitationsanalyse ein ganz eigenes Forschungsfeld erobert hat. Dieses Feld wird in vielen Wissenschaftsdisziplinen spätestens seit den 1950er Jahren intensiv beackert und auch in der US-amerikanischen Rechtswissenschaft seit knapp dreißig Jahren bestellt. Während Möglichkeiten und Grenzen der Zitationsanalyse international heftig diskutiert werden, nimmt die deutsche Jurisprudenz davon kaum Notiz. Dem soll der vorliegende Beitrag abhelfen.

\section{A. Zitationsanalyse: Jurisprudentias verlorene Tochter}

Mehr als in jeder anderen akademischen Disziplin geht es in der Rechtswissenschaft um kollektive Überzeugungsbildung (,h.M.“) durch wechselbezügliche Schriftwerke in Wissenschaft und Praxis. Daher überrascht es vielleicht nicht, dass es auch Juristen waren, die sich erstmals dafür interessierten, wer von wem wie oft zitiert wird. Das lässt sich spätestens um 1743 in England nachweisen und führte in den USA 1873 zur Gründung des berühmtesten Zitationsindex’ durch Frank Shepard. ${ }^{9}$ „Obwohl er aussieht wie ein mathematisches Tabellarium “, so wird der Index heute beschrieben, „ist Shepard's Citations ein Werkzeug von unschätzbarem Wert für die Suche nach Gerichtsentscheidungen, die sich früheren Präzedenzfällen anschließen, davon abgrenzen oder abweichen. “10 Für diese „Suche nach Gerichtsentscheidungen“ hat die US-amerikanische Rechtssprache sogar ein eigenes Verb: to shepardize.

4 T. Groh, Die Fußnote im Recht, in: J. Weidemann/A. Grub/pp., Festschrift für Forsen, Groh pp., Trier 1996, S. 73.

5 J. Basedow, Prolegomena zu einer funktionalistischen Theorie der Fußnote, ZEuP 2008, S. 671.

6 Groh, Fußnote (Fn. 4), S. 93.

7 Ausnahmen wohl einzig P. Häberle/A. Blankenagel, Fußnoten als Instrument der Rechts-Wissenschaft, Rechtstheorie 1988, S. 116; M. M. Siems, Wie häufig werden Zeitschriften und Kommentare zum BGB in der Literatur zitiert?, ZGS 2008, S. 90 m.w.N. in Fn. 3; Nachw. zu „allenfalls halbwissenschaftlichen" Vorarbeiten bei Groh, Fußnote (Fn. 4), S. 73.

8 Vgl. auch Basedow, Prolegomena (Fn. 5), S. 672: „Fußnotennachweise sind eine Form der Kommunikation mit Fachkollegen."; H. F. Moed, Citation Analysis, Springer, Dordrecht 2005, S. 26: "Citations or references can be conceived as social acts of members of the scholarly community.".

9 F. R. Shapiro, Origins of Bibliometrics, Citation Indexing, and Citation Analysis: The Neglected Legal Literature, 43 J. Am. Soc. Inf. Sci. 1992, S. 337 (338).

10 G. N. Hill/K. T. Hill, Shepardize, in: TheFreeDictionary, http://legal-dictionary.thefreedictionary.com/ Shepardize (Abruf am 1.12.2013, Übersetzung H.H.). 
Auch andere Fachdisziplinen begannen um die Wende des 20. Jahrhunderts mit der Ermittlung und Veröffentlichung von Zitationshäufigkeiten. ${ }^{11}$ Zum maßgeblichen Begründer der wissenschaftlichen Zitationsanalyse sollte jedoch der - später in Struktureller Linguistik promovierte - Eugene E. Garfield werden, der 1953 auf Shepard's Zitationsindex aufmerksam wurde,${ }^{12}$ kurz darauf erste Texte zur Entwicklung von Zitationsindizes für die Naturwissenschaften veröffentlichte ${ }^{13}$ und schließlich mit dem Institut für Wissenschaftsinformation (ISI) die bis heute weltweit maßgebliche Institution für naturwissenschaftliche Forschungsbibliographien gründete. Während die Zitationsanalyse in der US-amerikanischen Rechtswissenschaft auf das shepardizing beschränkt blieb, schossen in anderen Disziplinen neue Anwendungsmöglichkeiten der Zitationsanalyse wie Pilze aus dem Boden. Dazu gehören die Untersuchung von akademischen Netzwerken und wissenschaftlichen Entwicklungen ebenso wie die Einflussmessung einzelner Forschungsarbeiten oder Wissenschaftler (etwa durch den sog. „h-Index“ nach Hirsch) sowie ganzer Wissenschaftseinrichtungen oder Publikationsorgane (etwa durch den sog. „Impact Factor“). Mittlerweile ist die Literatur zum Thema schlicht unüberschaubar. ${ }^{14}$

Nachdem die Zitationsanalyse andere Disziplinen gut zwanzig Jahre lang auf den Kopf gestellt - und 1978 eine eigene Zeitschrift (Scientometrics) sowie 1979 eine monographische Aufarbeitung (Fn. 12) erhalten - hatte, kehrte sie in einer bemerkenswerten Wendung der Geschichte heim in den Schoß der Jurisprudenz. Das war das Verdienst des Rechtsbibliothekars Fred R. Shapiro, der später als „Gründungsvater" der „juristischen Zitierologie“ gefeiert wurde, ${ }^{15}$ weil er sich durch die Vorarbeiten Garfields dazu inspirieren ließ ${ }^{16}$ die Zitationsanalyse für juristische Fachaufsätze zu erproben. Seine 1985 erstmals veröffentlichte Liste der „meistzitierten Jura-Aufsätze“ stieß auf breites Interesse der juristischen Fachkreise, wurde 1996 grundlegend überarbeitet und zuletzt 2012 als Liste der „meistzitierten Jura-Aufsätze

11 L. Bornmann/H.-D. Daniel, What do citation counts measure?, 64 J. Docu. 2008, S. 45 zitieren eine Pionierarbeit von 1927; Shapiro, Origins (Fn. 9), S. 337 zitiert Arbeiten von 1917 bzw. 1874.

12 F. R. Shapiro, Origins (Fn. 9), S. 338 m.Verw. auf E. Garfield, Citation Indexing: Its Theory and Application in Science, Technology, and Humanities, John Wiley \& Sons, New York 1979, S. 6-18.

13 Vgl. E. Garfield, Citation Indexes for Science: A New Dimension in Documentation through Association of Ideas, 122 Science 1955, S. 108; später E. Garfield, Citation Indexing for Studying Science, 227 Nature 1970, S. 669; Garfield, Theory and Application (Fn. 12); weitere Nachw. unter http:// www.garfield.library.upenn.edu/pub.html.

14 Allein die Zeitschrift Scientometrics weist für die Suche nach "citation analysis" mehr als 2.500 Treffer aus. In den allgemeinen Wissenschaftszeitschriften Science und Nature vgl. v.a. Garfield (vorige Fn.); N. Wade, Citation Analysis: A New Tool for Science Administrators, 188 Science 1975, S. 429; G. Taubes, Measure for Measure in Science, 260 Science 1993, S. 884; D. Adam, Citation analysis: The counting house, 415 Nature 2002, S. 726.

15 J. M. Balkin/S. Levinson, How to Win Cites and Influence People, 71 Chi.-Kent L. Rev. 1996, S. 843: "Fred Shapiro can lay claim to be the founding father of a new and peculiar discipline: 'legal citology.'”; der Sammelband F. R. Shapiro, Collected Papers on Legal Citation Analysis, F. B. Rothman \& Co., Littleton 2001 versammelt Nachdrucke von vielen der nachfolgend erwähnten Studien.

16 Shapiro, Papers (Fn. 15), S. v. 
aller Zeiten " fortgesetzt. ${ }^{17}$ Diverse Ableger sind im Lauf der Zeit erschienen, ${ }^{18}$ bis hin zur Liste der meistzitierten Rechtswissenschaftler. ${ }^{19}$ Während sich Juristen zunächst mehr aus Neugier, Wettbewerbsmentalität und Sensationslust für diese Ranglisten interessiert haben mögen ("more because of human interest than profundity"), ${ }^{20}$ wurden stets auch ernsthafte Anwendungen diskutiert - befördert zunehmend durch die wiederholte Verbesserung der Methodologie seit 1985. Symposien zum Thema jagen einander seit fast zwanzig Jahren: von "Trends in Legal Citations and Scholarship" (1996) ${ }^{21}$ über "Empirical Evaluations of Specialized Law Reviews" $(1999)^{22}$ und "Interpreting Legal Citations" $(2000)^{23}$ bis hin zu "The Next Generation of Law School Rankings" (2006). ${ }^{24}$

Bis heute hat sich unter US-amerikanischen Juristen kein Konsens über Potential und Grenzen der Zitationsanalyse herausgebildet. Einer anfangs teils radikalen Ablehnung der gesamten Methodik ${ }^{25}$ folgten vermehrt entschiedene Befürworter ${ }^{26}$ sowie die obligatorische vermittelnde Ansicht. ${ }^{27}$ In Deutschland ist diese intensive Diskussion bisher gar nicht angekommen. Nur für die Rechtsprechung finden sich vereinzelt auch hierzulande theoretische Reflexionen ${ }^{28}$ und konkrete zitationsanalytische Stu-

17 F. R. Shapiro, The Most-Cited Law Review Articles, 73 Cal. L. Rev. 1985, S. 1540; F. R. Shapiro, The Most-Cited Law Review Articles Revisited, 71 Chi.-Kent L. Rev. 1996, S. 751; F. R. Shapiro/M. Pearse, The Most-Cited Law Review Articles Of All Time, 110 Mich. L. Rev. 2012, S. 1483.

18 F. R. Shapiro, The Most-Cited Articles from The Yale Law Journal, 100 Yale L. J. 1991, S. $1449 ;$ F. R. Shapiro, The Most-Cited Law Reviews, 29 J. Legal Stud. 2000, S. 389; F. R. Shapiro, 29 The MostCited Legal Books Published Since 1978, J. Legal Stud. 2000, S. 397.

19 F. R. Shapiro, The Most-Cited Legal Scholars, 29 J. Legal Stud. 2000, S. 409.

20 So Shapiro selbst, in: Papers (Fn. 15), S. v.

2116 Beiträge in: 71 Chi.-Kent L. Rev. 1996, S. 743 ff.

224 Beiträge in: 26 Fla. St. U. L. Rev. 1999, S. 813 ff.

2312 Beiträge in: 29 J. Legal Stud. 2000, S. $317 \mathrm{ff}$.

24 Min. 4 Beiträge in: 81 Ind. L. J. 2006, S. $1 \mathrm{ff}$.

25 Bspw. A. D. Austin, The Reliability Of Citation Counts In Judgments On Promotion, Tenure And Status, 35 Ariz. L. Rev. 1993, S. 829 (835): "Assume the purest of scholarly instincts and ideals. Assume an honest peer review system for law journals. In this untainted world, would cite counts be reliable indicators of scholarly influence? No, no, no.".

26 Bspw. Landes/R. A. Posner, Heavily Cited Articles In Law, 71 Chi.-Kent L. Rev. 1996, S. 825: "such studies can yield insights into both legal process and academic law and that they can also be useful tools for evaluating legal scholars (for purposes of promotions and prizes), judges, and even entire courts.".

27 Bspw. I. Ayres/F. Vars, Determinants Of Citations To Articles In Elite Law Reviews, 29 J. Legal Stud. 2000, 427 (430): "while there is a certain ineffable value to these studies, we wonder whether this value justifies the time and journal pages devoted to the subject.".

28 A. Nußberger, Wer zitiert wen? - Zur Funktion von Zitaten bei der Herausbildung gemeineuropäischen Verfassungsrechts, JZ 2006, S. 763; M. Jestaedt, Autorität und Zitat. Anmerkungen zur Zitierpraxis des Bundesverfassungsgerichts, in: S. Detterbeck/J. Rozek/C. v. Coelln, Recht als Medium der Staatlichkeit. Festschrift für Herbert Bethge zum 70. Geburtstag, Berlin 2009, S. 513. 
$\operatorname{dien}^{29}$ - nicht aber für Wissenschaftstexte, obwohl Nachbardisziplinen wie die Betriebswirtschaftslehre längst entsprechende Monographien erarbeitet ${ }^{30}$ und ein österreichischer Jurist das Potential der Zitationsanalyse für die kontinentalen Rechtsordnungen untersucht hat. ${ }^{31}$

Dabei ist auch deutschen Juristen durchaus bewusst, dass eine „empirische, mit quantitativen Methoden arbeitende Bestandsaufnahme von Fußnoten und Zitiergewohnheiten " wertvolle Erkenntnisse verspricht. ${ }^{32}$ Dass es an der konkreten Untersuchung und Erprobung dieser Methoden gleichwohl bis heute fehlt, mag deshalb weniger einer ablehnenden Grundhaltung als der fehlenden technischen Umsetzbarkeit geschuldet sein. Im Folgenden wird deshalb zunächst zu hinterfragen sein, welchen Mehrwert die Zitationsanalyse erwarten lässt und welchen Bedenken sie ausgesetzt ist (B. und C.). Sodann ist praktisch zu erproben, wie sich Zitationsanalyse umsetzen lässt (D.), um bisherige Berührungsängste abzubauen.

\section{B. Cui bono: Jahrmarkt der Eitelkeiten oder wissenschaftliche Erkenntnisquelle?}

Zitationsanalyse ist ein auf die Untersuchung von Nachweisapparaten beschränktes Teilgebiet der Bibliometrie, also der quantitativen Untersuchung von Schriftwerken. Soweit sich die Zitationsanalyse mit wissenschaftlichen Werken befasst, ist sie zugleich Teil der Szientometrie, also der Ausmessung wissenschaftlicher Diskurse. Zitationsanalyse will mess- und vergleichbar machen, was durch persönliche Eindrücke nur schwer beurteilt werden kann: Wissenschaftlicher Einfluss.

Indem Zitationsanalysen diesen Einfluss vergleichbar machen, könnte der Eindruck entstehen, es gehe dabei vordergründig um einen trivialen Wettstreit, der nur die Eitelkeit der daran beteiligten Forscher bediene, aber keinen nennenswerten Erkenntnisfortschritt ermögliche. So wird die Zitationsanalyse in den USA teilweise spitz karikiert:

"Citations analysis also unavoidably has a gossipy and at times tawdry aspect. We are drawn to citation rankings - reading and discussing them around the water cooler - but we are

29 R. Wagner-Döbler/L. Philipps, Präjudizien in der Rechtsprechung: Statistische Untersuchungen anhand der Zitierpraxis deutscher Gerichte, 23 Rechtstheorie 1992, S. 228; dies., Die Verbreitung neuer Rechtsbegriffe in der Rechtsprechung. Eine quantitative Untersuchung von Urteilstexten deutscher Gerichte 1950 bis 1992, 26 Rechtstheorie 1995, S. 235; R. Wagner-Döbler, Statistik der Entscheidungsgründe, JurPC 1994, S. 2454; ders., Präjudizien in deutschen, englischen und US-amerikanischen Gerichtsentscheidungen. Ein quantitativer Vergleich, 59 RabelsZ 1995, 113; W. Michel, Publikationsund Zitierverhalten bei Gerichtsentscheidungen, JurPC 1994, S. 2559; ders., Der Begriff der „herrschenden Meinung“ in der Rechtsprechung des BGH, JurPC 1995, S. 3010; ders., Häufigkeit von Zitaten des Bundesgesetzblattes in den Entscheidungen der Bundesgerichte, JurPC 1995, S. 3070.

30 C. Schmitz, Messung der Forschungsleistung in der Betriebswirtschaftslehre auf Basis der ISI-Zitationsindizes Köln 2008; S. Rassenhövel, Performancemessung im Hochschulbereich, Wiesbaden 2010.

31 A. Geist, Using Citation Analysis Techniques for Computer-Assisted Legal Research in Continental Jurisdictions, Edinburgh 2009, S. 66 ff. (online unter http://ssrn.com/abstract=1397674).

32 Häberle/Blankenagel, Fußnoten (Fn. 7), S. 117; Siems, Wie häufig (Fn. 7), S. 91. 
simultaneously repulsed by them criticizing their meanings (especially when we are excluded)."33

"More than a few of us will be eager to discover who ranks where and to speculate, with mixed tones of admiration, envy, and outright rancor, about the justice of whatever kudos are signified by high citation counts. [...] All law professors, no matter how famous, feel underappreciated, and hence all are interested in the most concrete symbols of appreciation. $" 34$

Diese Einwände sind nicht rundweg von der Hand zu weisen. Messbarkeit impliziert Vergleichbarkeit, und wo immer menschliche Leistungen miteinander verglichen werden können, ist der Wettstreit nicht weit. Deshalb erfreuen sich „Ranglisten aller Art“ in der Öffentlichkeit auch „nicht uneingeschränkter Beliebtheit“. ${ }^{35}$ Andererseits ist der Vergleich mit Standesgenossen die einzige Möglichkeit, sich ein realistisches Bild von der Welt zu machen, wo objektive Maßstäbe fehlen. ${ }^{36}$ In diesem Sinne eröffnet die Zitationsanalyse ganz neue Möglichkeiten der Forschungsevaluation, und zwar gleichermaßen bezogen auf Forscher, Publikationen und Wissenschaftseinrichtungen. ${ }^{37}$ Sie erleichtert nicht nur „Entscheidungen über Beförderung, Berufung und Status “, ${ }^{38}$ sondern wird auch unter den Vorzeichen der wachsenden Internationalisierung der Rechtswissenschaft, ihrer zunehmenden Abhängigkeit von Drittmitteln und dem Drang nach einer Auseinandersetzung mit überkommenen Modellen der Reputationsentwicklung relevant. ${ }^{39}$ Als weitere Hauptanwendungsfelder der Zitationsanalyse gelten die Verbesserung der Informationssuche in Datenbanken sowie die Modellierung von Diskursentwicklungen über die Zeit. ${ }^{40}$ So könnten Zitationsanalysen in der Rechtswissenschaft thematische Konjunkturen und intellektuelle Entwicklungen frühzeitig aufdecken, ${ }^{41}$ um sie kritisch hinterfragen und für die Praxis kommentieren zu können - ein wertvoller Beitrag zur juristischen Begriffs- und Prinzipienbildung.

33 Ayres/Vars, Determinants (Fn. 27), S. 447.

34 Balkin/Levinson, Win Cites (Fn. 15), S. $843 \mathrm{f}$.

35 M. Gröls/T. Gröls, Ein Ranking juristischer Fachzeitschriften, JZ 17/2009, Beil. S. 40, verfügbar unter http://www.mohr.de/fileadmin/user_upload/Zeitschriften/JZ/Ranking.pdf; H. Schulze-Fielitz, Staatsrechtslehre als Mikrokosmos, Tübingen 2013, S. 191: „methodisch und inhaltlich sehr problematisch und in der Bundesrepublik nicht üblich".

36 Sozialpsychologisches Gemeingut seit L. Festinger, A Theory of Social Comparison Processes 1954.

37 J. Nicolaisen, Citation Analysis, Annu. Rev. Inf. Sci. Tech. 2008, S. 609; ausf. Moed, Citation Analysis (Fn. 8).

38 So der Titel bei Austin, Reliability (Fn. 25).

39 Ausf. R. van Gestel/J. Vranken, Assessing Legal Research: Sense and Nonsense of Peer Review versus Bibliometrics and the Need for a European Approach, German Law Journal 2011, S. 901 (905 f.); Schulze-Fielitz, Staatsrechtslehre (Fn.35), S.187ff.; vgl. auch Wissenschaftsrat, Perspektiven der Rechtswissenschaft in Deutschland (Drs. 2558-12), Köln 2012 (online unter http://www.wissenschaftsrat.de/download/archiv/2558-12.pdf), S. 47.

40 Nicolaisen, Citation Analysis (Fn. 37), S. 609.

41 Vgl. Landes/Posner, Articles (Fn. 26), S. 829 ff. 
Gegen diese Art der Erkenntnisgewinnung wird eingewandt, dass es „in den Geisteswissenschaften problematisch“ sei, „,wissenschaftliche Qualität durch die Analyse der Zitierung der jeweiligen Publikation festzustellen“, weil „,die Häufigkeit der Zitierung in einer Fachzeitschrift [.] nicht ohne weiteres ein Beleg für wissenschaftliche Qualität" sei: ${ }^{42}$

„In der Rechtswissenschaft ist es möglich, durch Aufstellung einer abweichenden Meinung eine recht hohe Anzahl von Zitierungen zu erreichen, obne dass dies unbedingt einen Rückschluss auf die wissenschaftliche Qualität dieser abweichenden Meinung zulässt. "43

In die gleiche Stoßrichtung geht das häufig geäußerte Bedenken, dass Zitationsanalysen nicht zwischen zustimmenden und ablehnenden Zitierungen differenzieren, ${ }^{44}$ weshalb eine Veröffentlichung, die zehn Mal als abwegig zitiert wird, ebenso in die Wertung eingeht wie eine, die zehn Mal in höchsten Tönen gelobt wird. Was auf den ersten Blick als Manko erscheint, ist auf den zweiten allerdings völlig sachgerecht, denn jede ausdrückliche Ablehnung setzt ja voraus, dass das Abgelehnte sowohl wahr- als auch ernstgenommen wird: Es muss eine Replik „wert“ sein. Wer mag am wissenschaftlichen Wert der Werke von Werner Flume zweifeln, der der Nachwelt gerade mit dem Epithet „anderer Meinung “ in Erinnerung geblieben ist? ${ }^{45}$ Gerade weil die Rechtswissenschaft sich durch kollektive Meinungsbildung und fehlende objektive Maßstäbe auszeichnet, kann sie für „Qualität“ kaum ein anderes Maß angeben als die Fähigkeit, Widerspruch und Diskussion anzuregen:

"our notions of quality are not fully separable from notions of influence, not because influence necessarily follows quality as its just reward, but because disproportionate influence constructs our very notions of what good quality scholarship is." 46

Deshalb ist gerade für die Rechtswissenschaft, die keine außerhalb des Fachdiskurses stehenden Maßstäbe für „wissenschaftliche Qualität“ kennt, die Zitationsanalyse eine sachgerechte Herangehensweise. Natürlich dürfen quantifizierende Methoden nicht „als alleinige Bewertungsbasis, womöglich noch für die Einschätzung von individuellen Forschungsleistungen“ herhalten, denn das „gliche dem Versuch, die Qualität eines Restaurants einzig auf der Basis der Sauberkeit der sanitären Anlagen

42 P. Hanau/M. Gotthardt, Evaluation rechtswissenschaftlicher Forschung am Beispiel der Arbeitsrechtswissenschaft in Schweden, in: H. Konzen/S. Krebber/u.a., Festschrift für Rolf Birk, Tübingen 2008, S. 165 (173); ähnl. Wissenschaftsrat, Perspektiven (Fn. 39), S. 51.

43 Hanau/Gotthardt (vorige Fn.); A. L. Brophy, The Signaling Value Of Law Reviews, 36 Fla. St. U. L. Rev. 2009, S. 229 (233): "citations are a measure of popularity of sorts. Yet, popularity is not always a measure of quality.”.

44 Gröls/Gröls, Ranking (Fn. 35), S. 32: „So mag ein Artikel als kontrovers oder gar fehlerhaft wahrgenommen und in der Folge häufig aufgegriffen werden - für die Qualität dieses Artikels spricht dies dann jedoch gerade nicht.“; ähnl. Wissenschaftsrat, Perspektiven (Fn. 39), S. 51: Es gehöre „zur rechtswissenschaftlichen Zitationspraxis, falsche Gedanken oder abweichende Meinungen [...] anzuführen"; dag. aber Siems, Wie häufig (Fn. 7), S. 91.

45 Vgl. nur den Titel bei H. Prantl, ... anderer Meinung: Flume!, Süddt. Ztg. v. 12.9.2003.

46 Balkin/Levinson, Win Cites (Fn. 15), S. 844; ähnl. Schulze-Fielitz, Staatsrechtslehre (Fn. 35), S. 200 zur „Unklarheit der sachlichen Kriterien für [wissenschaftliche] Reputation“. 
bewerten zu wollen “. ${ }^{47}$ Ein ergänzender und angemessen kontextualisierter Einsatz der Zitationsanalyse hingegen wird auch von Kritikern nicht grundsätzlich abgelehnt:

„In der Evaluationswissenschaft wird deshalb von herrschenden [sic] Meinung eine Kombination von, peer review" und der Anwendung von Wissenschaftsindikatoren verlangt. "48

Es ist also davon auszugehen, dass die Zitationsanalyse Juristen dabei helfen kann, ihre subjektiven Eindrücke und Vorurteile zu überprüfen, wodurch sie zur Evidenzbasierung der Rechtswissenschaft beiträgt. ${ }^{49}$ Diese abstrakte Behauptung sei an konkreten Untersuchungsfragen aus US-amerikanischen Studien der letzten Jahre illustriert:

- Nimmt die Rechtsprechung akademische Veröffentlichungen wahr oder sind diese eher praxisfern? ${ }^{50}$

- Erhöht die digitale Verfügbarkeit von Volltextdatenbanken die Wahrnehmung älterer Fachaufsätze? ${ }^{51}$ Oder die Wahrnehmung weniger renommierter Zeitschriften? 52

- Erhöhen Ruf und Ansehen einer Zeitschrift überhaupt die Wahrnehmung der darin veröffentlichten Texte?53

- Wie wichtig ist die Reihenfolge der Aufsätze innerhalb eines Zeitschriftenhefts? ${ }^{54}$

- Sind etablierte Autoren produktiver als ihre jüngeren Kollegen?55

47 Gröls/Gröls, Ranking (Fn. 35), S. 40.

48 Hanau/Gotthardt, Evaluation (Fn. 42), S. 168; auch nach Gröls/Gröls, Ranking (Fn. 35), S. 40 können quantitative Methoden „nur ein Mosaikstein sein, wenn es etwa darum geht, Forschungseinrichtungen und deren Personal zu bewerten "; krit. Van Gestel/Vranken, Research (Fn. 39), S. 921: "A cumulative implementation of peer review and metrics will perhaps swell bureaucracy to an unacceptable level.".

49 Dazu ausf. H. Hamann, Evidenzbasierte Jurisprudenz. Methoden empirischer Forschung und ihr Erkenntniswert für das Recht am Beispiel des Gesellschaftsrechts, Tübingen 2014.

50 Ersteres, laut M. M. Harner/J. A. Cantone, Is Legal Scholarship Out Of Touch?, 19 U. Miami Bus. L. Rev. 2011, S. 1; die Pointe dieser Erkenntnis liegt in der US-spezifischen Wahrnehmung der Rechtswissenschaft als besonders praxisfern (s.u. bei Fn. 117).

51 Nein, laut M. S. Lowe/K. L. Wallace, HeinOnline and Law Review Citation Patterns, 103 Law Libr. J. 2011, S. 55 (67f.).

52 Ja, laut D. J. Callahan/N. Devins, Law Review Article Placement: Benefit or Beauty Prize?, 56 J. Legal Edu. 2006, S. 374 (387).

53 Kaum, laut Brophy, Signaling (Fn. 43), S. 229; vgl. auch Callahan/Devins, Placement (Fn. 52); dagegen Schulze-Fielitz, Staatsrechtslehre (Fn. 35), S. 190, 192: „vermutlich ist beispielsweise ein Aufsatz im Archiv des öffentlichen Rechts wissenschaftlich lohnender [... und] lässt ein anderes psychologisches Gewicht vermuten als etwa in den Juristischen Arbeitsblättern oder in ,Der Landkreis‘.“.

54 Die Erstplatzierung im Heft verdreifacht die Zitationen, laut T. Coupe/V. Ginsburgh/A. Noury, Are leading papers of better quality? Evidence from a natural experiment, 62 Ox. Econ. Papers 2010, S. 1 (6): "two thirds of the additional cites that leading papers get seem to be due to the effect of going first” (für die Eur. Econ. Rev. 1975-97); dazu Schulze-Fielitz, Staatsrechtslehre (Fn. 35), S. 192: „auch die Reihenfolge der Autoren nach dem ,Spitzenaufsatz' in den einzelnen Ausgaben der Zeitschriften spiegelt in der Regel Reputationsdifferenzen “.

$55 \mathrm{Ja}$, aber nur in klassischen Rechtsgebieten, laut Landes/Posner, Articles (Fn. 26), S. 832 ff. 
- Wie oft werden Veröffentlichungen blind zitiert und Fundstellen direkt abgeschrieben? ${ }^{56}$

Auch ganz praktische Anliegen ${ }^{57}$ sowie die „bloße“ Rechtstatsachenforschung, die einer analytischen Aufarbeitung oft vorausgeht, können von der Zitationsanalyse profitieren. Zwei kurze Auszüge aus Studien US-amerikanischer Juristen mögen dies belegen:

"To summarize a few of our results: citations per year peak at 4 years after publication, and an article receives half of its expected total lifetime citations after 4.6 years; appearing first in an issue is a significant advantage; international law articles receive fewer citations; jurisprudence articles are cited more often; articles by young, female, or minority authors are more heavily cited. Articles with shorter titles, fewer footnotes per page, and without equations have significantly more citations than other articles. Total citations generally increase with an article's length, but citations per published page peak at 53 pages." 58

"we find that lateral hires outperform entry-level hires, even when controlling for years in teaching, though not at every school. We find no strong evidence that citation performance correlates with sex once one controls for years in teaching. We find evidence that non-minority males in teaching fewer than 8 years have higher citations rates than other groups. This effect fades for those in teaching more than 7 years." 59

Ähnliche Erkenntnisse über die Wissensstruktur und Forschungslandschaft der deutschen Rechtswissenschaft wären praktisch ebenso nützlich wie theoretisch relevant.

\section{Motivbündel: Eine kommunikationspsychologische Theorie der Fußnote}

Von den zahlreichen Einwänden, denen sich die Zitationsanalyse in der US-amerikanischen Rechtswissenschaft seit ihren Anfängen ausgesetzt sieht, ${ }^{60}$ beruhen viele auf Eigenheiten der US-amerikanischen Rechtskultur und Wissenschaftsstruktur. ${ }^{61}$

56 In 70-90\% der Fälle, laut M. V. Simkin/V. P. Roychowdhury, Stochastic modeling of citation slips, 62 Scientometrics 2005, S. 367; dies., Read Before You Cite!, Compl. Syst. 2003, S. 269 (jeweils für die Physik); ähnl. M. Wright/J. S. Armstrong, Verification of Citations: Fawlty Towers of Knowledge?, 38 Interfaces 2008, S. 125 (für die BWL).

57 Etwa die Frage von K. C. Brown, How Many Copies Are Enough? Using Citation Studies to Limit Journal Holdings, 94 Law Libr. J. 2002, S. 301.

58 Ayres/Vars, Determinants (Fn. 27), S. 427.

59 T. Eisenberg/M. T. Wells, Ranking and Explaining the Scholarly Impact Of Law Schools, 27 J. Legal Stud. 1998, S. 373 (413).

60 Dazu bspw. A. D. Austin, Footnotes as Product Differentiation, 40 Vand. L. Rev. 1987, S. $1131 ;$ A. D. Austin, Footnote Skullduggery and Other Bad Habits, 44 U. Miami L. Rev. 1990, S. 1009; A. D. Austin, Political Correctness is a Footnote, 71 Or. L. Rev. 1992, S. 543; Austin, Reliability (Fn. 25), S. 829; Balkin/Levinson, Win Cites (Fn. 15), S. 843; knappe Zusammenfassung disziplinübergreifender Einwände bei M. H. MacRoberts/B. R. MacRoberts, Problems of Citation Analysis, 36 Scientometrics 1996, S. 435.

61 Bspw. sieht Austin, Reliability (Fn. 25), S. 832 f. Hindernisse für die Zitationsanalyse in der Vorherrschaft studentischer Rechtszeitschriften ("Student editors are more interested in playing mind games with the latest edition of the Bluebook than looking at the relevance and content of cites."), und der zunehmenden "politicization of legal education and writing", die in Deutschland in dieser Form nicht stattfindet. Ebenso an den deutschen Verhältnissen vorbei geht die Kritik von Balkin/Levinson, Win Cites (Fn. 15), S. 843, dass ein Großteil der juristischen Aufsätze völlig praxisfern sei (864: "The Real World? What's that?") und von Studenten stamme (860: "much of the writing that appears in law journals is not by law professors at all. It is by students.”). 
Andere mögen auf die deutschen Gegebenheiten übertragbar sein, allerdings fehlt insoweit bislang jegliche Auseinandersetzung. Hier sei deshalb nur ein Einwand untersucht, der in den USA sehr prominent ist und auf die Gegebenheiten der deutschen Rechtswissenschaft uneingeschränkt übertragbar sein dürfte. Dabei wird sich herausstellen, dass dieser Einwand das Unternehmen der Zitationsanalyse nicht grundsätzlich in Frage stellt, sondern in dieses vielmehr theoretisch integriert werden kann. Dadurch trägt der vorliegende Beitrag auch zum theoretischen Grundgerüst der $\mathrm{Zi}$ tationsanalyse bei, die zwar zwei herrschende Schulen ${ }^{62}$ und originelle Ableger ${ }^{63}$ hervorgebracht, aber bis heute keine einheitliche Theorie gefunden hat.

Der Einwand, von dem die Rede ist, setzt noch grundsätzlicher an als die bereits erörterte Kritik an der Disparität von „Einfluss“ und „Qualität“: Belegen Zitationen überhaupt den Einfluss wissenschaftlicher Ideen? Schließlich sind im sozialen Miteinander der (Rechts)Wissenschaft ein ganzes Bündel anderer Motive denkbar:

"Without a screening system, determination of an author's citation motivation is impossible. Ideology, academic politics, fun, sycophancy - any motivation is possible." 64

Gerade für die deutsche Rechtswissenschaft werden ganz unterschiedliche Intentionen für die Verwendung von Fußnoten identifiziert: Beleg-, Selbstreferenz-, Verweis-, Explikations-, Digressions-, Kommentierungs- und Metafußnoten ${ }^{65}$ werden unterschieden, ebenso wie Alibi-, Ornamental-, Nachweis-, Gelehrsamkeits-, Zweitabhandlungs-, Streit- oder Schmeichel- und Reserve-Fußnoten. ${ }^{66}$ Diese implizite Bündelung ganz unterschiedlicher Zitationsmotive könnte die Grundfesten der Zitationsanalyse erschüttern und deren Sinn insgesamt infrage stellen:

"the difficulty of identifying the true meaning of many or few citations is so endemic that to our minds it calls into question the entire enterprise." 67

So berechtigt und schwerwiegend dieser Einwand allerdings sein mag, so wenig sollte er dazu führen, die Flinte voreilig ins Korn zu werfen. Natürlich haben Fußnoten als eine Form der menschlichen Kommunikation (vgl. oben bei und in Fn. 8) an der Vieldeutigkeit und den Unwägbarkeiten jeder menschlichen Kommunikation teil. Das rechtfertigt aber keinen Verzicht auf die Auseinandersetzung mit Kommunikationshandlungen, sondern erfordert gerade ein analytisches Herangehen. Immerhin steht für die Analyse von Motivbündeln in jeder Form des „miteinander redens“

62 Bornmann/Daniel, Measure (Fn.11), S. 48 ff.; Nicolaisen, Citation Analysis (Fn. 37), S. 615 ff.; MacRoberts/MacRoberts, Problems (Fn. 60), S. 438 ff.

63 Z.B. Nicolaisen, Citation Analysis (Fn. 37), S. 624 ff. mit Anleihen aus der Evolutionsbiologie.

64 Austin, Reliability (Fn. 25), S. 832; Schulze-Fielitz, Staatsrechtslehre (Fn. 35), S. 333: „Sekundärmotive"; blumiger formuliert bei Schwanitz, Bildung (Fn. 3).

65 Groh, Fußnote (Fn. 4), S. 78 ff.

66 Basedow, Prolegomena (Fn. 5), S. 671 f.; Schulze-Fielitz, Staatsrechtslehre (Fn. 35), S. 197, 336 spricht gar von „Zitier- und Lobekartellen“.

67 Ayres/Vars, Determinants (Fn. 27), S. 430. 
bereits eine ausgearbeitete Theorie zur Verfügung. ${ }^{68}$ Danach transportiert menschliche Kommunikation stets Botschaften auf mindestens vier unterscheidbaren Ebenen, nämlich der Sachebene („Es ist...“), der Selbstoffenbarungsebene („Ich bin...“), der Beziehungsebene („Du bist...“) und der Appellebene („Du sollst...“);69 zur Beziehungsebene gehören auch Fragen der Beziehungsdefinition („Wir sind...“), ${ }^{70}$ die Selbstoffenbarungsebene zerfällt in die bewusste Selbstdarstellung und die unbewusste Selbstenthüllung. ${ }^{71}$ Insgesamt ergeben sich also sechs analytische Kategorien.

Betrachtet man Fußnoten aus diesem Blickwinkel heraus, wird deutlich, dass sie nicht nur eine Sachinformation enthalten (und damit eine Aussage über wissenschaftlichen Einfluss zulassen), sondern dass sie zugleich mindestens fünf andere Botschaften transportieren, wobei die Schwerpunktsetzung je nach den Umständen variieren kann. So könnte etwa die Fußnote

Ebenso Heck, AcP 112 (1914) 1.

mindestens sechserlei bedeuten:

- Sachinformation: „Der formulierte Gedanke findet sich ebenso bei Heck.“

- Selbstdarstellung: „Ich, Autor, habe Heck gelesen und verstanden.“72

- Selbstenthüllung: „Der Autor muss offenbar seinen Selbstwert erhöhen, indem er Heck zitiert."

- Fremddarstellung: „Du, Leser, wusstest bisher nicht, wo du das nachlesen kannst."

- Beziehungsdefinition: „Du, Leser, hast mir, Autor, gegenüber ein Anrecht auf diesen Nachweis."

- Appell: „Du, Leser, solltest dich wirklich mal mit Heck beschäftigen.“73

Diese sechs Kategorien dürften genügen, um alle oben (bei Fn. 65, 66) genannten Fußnotenarten zu erfassen. ${ }^{74}$ Damit steht ein theoretisches Grundgerüst zur Verfügung, um kommunikative Motivbündel bei der Fußnotenverwendung analytisch zu erschließen, statt davor zu kapitulieren.

68 Laienverständlich erläutert in: F. Schulz von Thun, Miteinander Reden, Bd. 1: Störungen und Klärungen. Allgemeine Psychologie der Kommunikation, 48. Aufl., Reinbek 2010.

69 Von Thun, Reden (vorige Fn.), S. 26-30; allgemeiner unterfallen diese vier Ebenen dem „Ausdrucks-“ oder dem "Wirkungsaspekt“" (ebd., S. 209 ff.).

70 Von Thun, Reden (Fn. 68), S. 28.

71 Von Thun, Reden (Fn. 68), S. 27.

72 Vgl. Balkin/Levinson, Win Cites (Fn. 15), S. 867: "Citations, we think, are often just [...] a way of displaying information the citing author wants to convey about him- or herself, while concealing other information that would interfere with his or her desired 'performance' as a legal scholar.".

73 Vgl. Balkin/Levinson, Win Cites (Fn. 15), S. 844: "Citations and quotations not only draw attention to works, they make works the kinds of works that attention is paid to and hence should be paid to.".

74 Bspw. setzen „Gelehrsamkeitsfußnoten“ den Schwerpunkt auf die Selbstdarstellung, "Streit- und Schmeichelfußnoten" dagegen auf die Beziehungsdefinition. 
Diese kommunikationspsychologische Betrachtung der Fußnote ermöglicht es, bei der Zitationsanalyse nach angemessenen Operationalisierungen für die unterschiedlichen Kommunikationsebenen zu suchen. Solange diese noch nicht zur Verfügung stehen, hilft eine zusätzliche Überlegung: Motivbündel können die Zitationsanalyse überhaupt nur dann ernsthaft behindern, wenn alle denkbaren Motive gleichermaßen willkürlich auftreten. Gerade das ist aber nicht der Fall: Zwar bespielen Fußnoten unweigerlich mehrere Kommunikationsebenen, doch ihr einziger durchweg als legitim anerkannter Zweck ist jener der Sachinformation. So sehr ein Autor Gelehrsamkeit zur Schau stellen, Streitigkeiten austragen oder sonst einen sozialen Zweck verfolgen will, so sehr muss er seine Fußnote an den Haupttext rückbinden, also zumindest auch eine Sachinformation verpacken. Damit ist die Sachebene die einzige „Konstante“ bei der Fußnotenverwendung. Andere Motive mögen ein starkes Hintergrundrauschen bilden, bleiben aber mangels Regelhaftigkeit ihres Gebrauchs zufallsbestimmt. Damit sind sie in der Sprache der empirischen Forschung ganz gewöhnliche Störvariablen, deren Auswirkungen sich dank des Gesetzes der großen Zahl statistisch herausrechnen lassen. Denn Zweck der quantitativ-empirischen Forschung ist ja gerade, robuste Regelmäßigkeiten in großen Datenmengen freizulegen. ${ }^{75}$

\section{Praktische Erprobung: Pilotstudien zur Zitationsanalyse}

Um zuletzt das Potential und die Grenzen der Zitationsanalyse für die deutsche Rechtswissenschaft konkret zu erkunden, bedarf es geeigneter Daten (dazu I.), die sodann aufbereitet (II.) und im dritten Schritt ausgewertet werden können (III.).

\section{Datenerhebung}

Eine Zitationsanalyse benötigt Nachweisapparate in digitalem Format. Dazu lassen sich entweder - zeitaufwändig und fehleranfällig - die Fußnoten gedruckter Medien abtippen, oder diejenigen digitalen Inhalte nutzen, die von Verlagen zur Verfügung gestellt werden und dem Inhalt der zugehörigen Druckveröffentlichung entsprechen (sollten). Solche Datenbanken stecken in der deutschen Rechtswissenschaft allerdings noch in den Anfängen. Zwar liegen erste Kommentare und Monographien bereits digital vor; die größten Fortschritte allerdings hat die Digitalisierung bislang bei den Zeitschriften gemacht. Da viele Zeitschriften mittlerweile gar nicht mehr in ihrer Druckfassung, sondern direkt in digitalen Datenbanken gelesen und zitiert werden, empfiehlt sich die Nutzung dieser Datenbanken auch für die Zitationsanalyse. Dabei lassen sich allerdings nur solche Datenbanken verwenden, die digitale Volltexte vorhalten und nicht nur Faksimiles im pdf-Format.

75 Hamann, Jurisprudenz (Fn. 49), S. 77 ff. m.w.N.; vgl. auch Siems, Wie häufig (Fn. 7), S. 90. 
Weil bei weitem noch nicht alle juristischen Zeitschriften digital verfügbar sind und diese Verfügbarkeit von zahlreichen Faktoren abhängt, muss sich die Zitationsanalyse zwar auf eine sog. Gelegenheitsstichprobe beschränken und kann keine „repräsentativen " Ergebnisse erbringen. Zugleich sind aber immerhin so viele Zeitschriften digitalisiert, dass ein erheblicher Teil des Diskurses untersucht werden kann und sogar eine gezielte Auswahl erforderlich ist, um die Materialmenge überhaupt bewältigen zu können. Für diese Auswahl wurden die folgenden Kriterien festgelegt:

- Die untersuchten Zeitschriften sollten weithin bekannt, also mutmaßlich diskursrelevant sein.

- Die untersuchten Zeitschriften sollten sowohl Ausbildungs-, Praktiker- als auch wissenschaftliche Archivzeitschriften umfassen.

- Die untersuchten Zeitschriften sollten alle Rechtsgebiete - Bürgerliches, Öffentliches und Strafrecht - abdecken, um Unterschiede zwischen den Gebieten erkennen zu können.

- Zugleich sollten aber hinreichend viele Zeitschriften ein und dem selben Fachgebiet zugehören, um unterschiedliche Medien mit gemeinsamen Themen zu erfassen.

Nach diesen Kriterien wurden die folgenden vierzehn Zeitschriften ausgewählt, für die jeweils mindestens acht Jahrgänge digital vorliegen:

\begin{tabular}{|l|l|c|c|c|}
\hline Kürzel & Zeitschriftenname & Zeitraum & $\begin{array}{c}\text { Jahr- } \\
\text { gänge }\end{array}$ & Datenbank \\
\hline AG & Die Aktiengesellschaft & $1991-2012$ & 22 & juris \\
\hline BB & Betriebs-Berater (inkl. Beilage) & $1991-2012$ & 22 & juris \\
\hline DZWIR & Deutsche Zeitschrift für Wirtschafts- u. InsolvenzR & $2002-2012$ & 11 & juris \\
\hline JA & Juristische Arbeitsblätter & $2005-2012$ & 8 & BeckOnline \\
\hline JuS & Juristische Schulung & $2000-2012$ & 13 & BeckOnline \\
\hline MDR & Monatsschrift für Deutsches Recht & $1981-2012$ & 32 & wiso \\
\hline NJW & Neue Juristische Wochenschrift (inkl. Beilage) & $1981-2012$ & 32 & BeckOnline \\
\hline NStZ & Neue Zeitschrift für Strafrecht & $1981-2012$ & 32 & BeckOnline \\
\hline NVwZ & Neue Zeitschrift für Verwaltungsrecht (inkl. Beil.) & $1982-2012$ & 31 & BeckOnline \\
\hline NZG & Neue Zeitschrift für Gesellschaftsrecht & $1998-2012$ & 15 & BeckOnline \\
\hline NZM & Neue Zeitschrift für Miet- u. Wohnungsrecht & $1998-2012$ & 15 & BeckOnline \\
\hline ZGR & Zeitschrift für Unternehmens- u. Gesellschaftsrecht & $2000-2012$ & 13 & juris \\
\hline ZIP & Zeitschrift für Wirtschaftsrecht & $1980-2012$ & 33 & ZIP online \\
\hline ZRP & Zeitschrift für Rechtspolitik & $2000-2012$ & 13 & BeckOnline \\
\hline
\end{tabular}

Tab. 1: Rabmendaten des für die Zitationsanalyse verwendeten Zeitschriftenbestands

76 Die unterschiedliche Länge der Zeiträume (sog. unbalanced panel) könnte zu Verzerrungen führen, wenn sich das Zitierverhalten im Lauf der Zeit verändert hätte. Erste Tests bieten für solche Zeiteffekte keinen Anhaltspunkt, genauere Untersuchungen müssen späteren Arbeiten vorbehalten bleiben. 
Diese Zeitschriften umfassen sowohl allgemeine Zeitschriften (JA, JuS, MDR, NJW, ZRP) als auch solche aus dem Strafrecht (NStZ) und öffentlichen Recht (NVwZ) sowie aus dem besonderen Schuldrecht (NZM). Der Schwerpunkt liegt indessen klar auf dem Wirtschaftsrecht (AG, BB, DZWIR, NZG, ZGR, ZIP). Die meisten Zeitschriften lassen sich als Praktikerzeitschriften charakterisieren, mit Ausnahme der beiden Ausbildungszeitschriften JA und JuS sowie der Archivzeitschrift ZGR. Bedauerlicherweise scheint noch keine andere Archivzeitschrift im digitalen Volltext vorzuliegen, obwohl bspw. die Archivzeitschriften des Verlags Mohr Siebeck (AcP, AöR, etc.) sowie dessen renommierte „JuristenZeitung“ (JZ) teilweise für den gesamten Zeitraum ihres Erscheinens im pdf-Format digital faksimiliert sind.

Die ausgewählten Zeitschriften enthalten sehr unterschiedliche Textarten (Rechtsprechung, Anmerkungen, Rezensionen, Aufsätze, etc.), deren Zitationspraktiken weder im „ob“ noch im „wie“ übereinstimmen. Zugunsten der Vergleichbarkeit beschränkte sich die Zitationsanalyse deshalb auf das eine Medienformat, in dem Zitationen vermutlich die größte Rolle spielen: Texte, die in der jeweiligen Datenbank als „Aufsatz“ gekennzeichnet waren. Das umfasste in den 14 Zeitschriften insgesamt 34.334 Texte, also durchschnittlich 118 Aufsätze je Zeitschrift und Jahr; genauere Aufschlüsselungen sind der folgenden Tabelle zu entnehmen.

Alle Aufsätze zusammen enthielten 1.671.243 Fußnoten, ${ }^{77}$ aus denen sodann für einen ersten statistischen Überblick „die wesentlichen Werte“ berechnet wurden, „die einer empirischen Erfassung dienlich sind“: ${ }^{78}$

77 Dabei wurden 22.050 nicht-numerische Fußnoten (z.B. *) ignoriert, da sie überwiegend nur Autorenangaben enthalten - wenngleich einzelne Texte bis zu fün *-Fußnoten aufweisen, darunter auch solche mit bibliographischen Angaben (z.B. Serick, BB 1995, 2013).

78 Groh, Fußnote (Fn. 4), S. 91, auf S. 92 f. mit der Trias Fußnotendichte, Fußnotenfrequenz, Fußnotenquote. 


\begin{tabular}{|c|c|c|c|c|c|c|}
\hline \multirow[b]{2}{*}{ Zeitschrift } & \multicolumn{2}{|c|}{ Anzahl der Aufsätze } & \multicolumn{4}{|c|}{ Nur für Aufsätze mit Fn.: durchschnittliche(r) Fußnoten- } \\
\hline & insgesamt & mit Fn. & -anzahl ${ }^{79}$ & -länge $e^{80}$ & - dichte $^{81}$ & -anteil ${ }^{82}$ \\
\hline AG & 1.140 & $94,47 \%$ & 74,58 & 169,95 & $1 / 807$ & $21,39 \%$ \\
\hline $\mathrm{BB}$ & 7.096 & $94,17 \%$ & 45,68 & 126,99 & $1 / 1.074$ & $15,03 \%$ \\
\hline DZWIR & 364 & $98,35 \%$ & 55,61 & 117,59 & $1 / 778$ & $16,55 \%$ \\
\hline JA & 1.020 & $68,63 \%$ & 56,62 & 115,24 & $1 / 1.035$ & $17,35 \%$ \\
\hline JuS & 2.241 & $95,27 \%$ & 44,37 & 133,86 & $1 / 861$ & $17,00 \%$ \\
\hline MDR & 2.071 & $90,44 \%$ & 41,16 & 115,54 & $1 / 901$ & $16,61 \%$ \\
\hline NJW & 8.235 & $95,57 \%$ & 54,14 & 117,95 & $1 / 863$ & $16,41 \%$ \\
\hline NStZ & 1.953 & $86,84 \%$ & 49,65 & 129,92 & $1 / 1.428$ & $16,53 \%$ \\
\hline NVwZ & 3.256 & $99,14 \%$ & 54,63 & 126,96 & ${ }^{1} / 757$ & $17,85 \%$ \\
\hline NZG & 1.569 & $98,34 \%$ & 51,45 & 129,74 & $1 / 730$ & $18,75 \%$ \\
\hline NZM & 1.018 & $98,43 \%$ & 45,96 & 104,05 & $1 / 1.071$ & $14,10 \%$ \\
\hline ZGR & 441 & $91,61 \%$ & 112,88 & 157,38 & $1 / 900$ & $21,82 \%$ \\
\hline ZIP & 2.831 & $97,81 \%$ & 60,19 & 138,00 & $1 / 817$ & $18,30 \%$ \\
\hline ZRP & 1.099 & $90,90 \%$ & 30,02 & 121,36 & $1 / 1.216$ & $14,58 \%$ \\
\hline Insgesamt & 34.334 & $94,18 \%$ & 51,68 & 126,37 & $1 / 936$ & $16,72 \%$ \\
\hline
\end{tabular}

Tab. 2: Aufschlïsselung relevanter Parameter des verwendeten Textbestands

Schon anhand dieser Tabelle werden unterschiedliche Zitationspraktiken unter den Zeitschriften sichtbar. Einige bemerkenswerte Unterschiede sind fett hervorgehoben: Während in den meisten Zeitschriften $90 \%$ und mehr der Aufsätze über Fußnoten verfügen, liegen die NStZ und die JA darunter. Bei der durchschnittlichen Anzahl der Fußnoten führt mit weitem Abstand die einzige Archivzeitschrift des untersuchten Bestands (ZGR), die größte durchschnittliche Fußnotenlänge dagegen weist eine Zeitschrift auf, von der das vielleicht nicht auf den ersten Blick zu vermuten gewesen wäre (AG), gefolgt wiederum von der ZGR. Relativ zum Haupttext verfügt die NZG über die größte Fußnotenzahl, während das Schlusslicht - wiederum die NStZ - nur halb so viele Fußnoten je gegebener Textlänge enthält. Setzt man nicht die Anzahl, sondern die Länge der Fußnoten ins Verhältnis zum Haupttext, führen wiederum ZGR und AG die Liste an.

79 Die meisten Fußnoten hatten die Beiträge von Bron, BB-Beil. 7/2007, 2 (467 Fn.); Emde, BB-Beil. 3/2007, 3 (450 Fn.); Sonnenschein, NJW 1998, 2172 (431 Fn.).

80 Angegeben in Zeichen. Die längsten Fußnoten waren: Herz, JA 2009, 573 Fn. 101 (5.887 Zeichen); Meyer/Hetzer, NJW 1998, 1017 Fn. 6 (5.836 Zeichen); H. W. Schmidt, MDR 1984, 969 Fn. 26 (5.651 Zeichen).

81 Anzahl der Fußnoten relativ zur Länge des Haupttextes. „1/936 “ bedeutet: Im Durchschnitt folgt auf je 936 Zeichen des Haupttextes eine Fußnote, oder anders: Ein Text von 9.360 Zeichen enthält durchschnittlich 10 Fußnoten.

82 Anteil der Fußnoten an der gesamten Textmenge. „16 \% “ bedeutet: Von 100 Zeichen entfallen 84 auf den Haupttext, 16 auf die Fußnoten, oder anders: Alle Fußnoten zusammen sind knapp ein Fünftel $(16 / 84)$ so lang wie der Haupttext. 
So interessant diese Rahmenparameter für sich genommen bereits sein mögen, bedarf die eigentliche Zitationsanalyse im nächsten Schritt einer weiterführenden Datenaufbereitung.

\section{Datenaufbereitung}

Ziel der anschließenden Datenaufbereitung war es, aus jeder dieser Fußnoten die zitierten Fundstellen automatisiert zu erheben. ${ }^{83}$ Automatisierte Erhebung setzt ein hohes Maß an Standardisierung voraus, das im juristischen Schrifttum vor allem für die Zitierung von Zeitschriften erreicht wird. Denn jede Zeitschrift wird mit einem ganz bestimmten, nahezu eindeutigen Kürzel zitiert. ${ }^{84}$ Um eine vollständige Liste dieser Zeitschriftenkürzel zu erhalten, konnte auf drei umfangreiche Verzeichnisse zurückgegriffen werden:

\begin{tabular}{|l|l|l|c|}
\hline Anbieter & Name & URL & Kürzel \\
\hline juris & $\begin{array}{l}\text { Verzeichnis der ausgewerteten juristischen } \\
\text { Periodika für die Datenbank Aufsätze }\end{array}$ & $\begin{array}{l}\text { www.juris.de/jportal/nav/unter- } \\
\text { nehmen/auswertung/fachzeit- } \\
\text { schriftenaufsaetze.jsp }\end{array}$ & 797 \\
\hline Kuselit & $\begin{array}{l}\text { Gesamtliste der von Kuselit dokumentier- } \\
\text { ten Zeitschriften }\end{array}$ & $\begin{array}{l}\text { www.kuselit.de/cms/index.php? } \\
\text { folder=1041\&op=gesamtliste }\end{array}$ & 738 \\
\hline C.H. Beck & $\begin{array}{l}\text { Karlsruher Juristische Bibliographie: Ver- } \\
\text { zeichnis der für Periodika verwendeten } \\
\text { Abkürzungen }\end{array}$ & $\begin{array}{l}\text { rsw.beck.de/rsw/upload/KJB/KJB- } \\
\text { ZS-Abkuerz.htm }\end{array}$ & 497 \\
\hline
\end{tabular}

Tab. 3: Verwendete Zeitschriftenverzeichnisse mit der jeweils enthaltenen Anzahl Zeitschriftenkürzel

Die in diesen Verzeichnissen nachgewiesenen Zeitschriftenkürzel wurden Ende Januar 2013 abgerufen und in eine konsolidierte Gesamtliste von 1.371 Kürzeln zusammengeführt.

Im nächsten Schritt waren alle „Fundstellen“ in den vorliegend ausgewerteten Zeitschriften zu identifizieren. Fundstellen kombinieren ein Zeitschriftenkürzel mit weiteren Angaben, wobei sie fast durchweg einer von drei Konventionen folgen:

- Archivzeitschriften werden so zitiert, dass auf den Autor des Textes meist ein Komma folgt, darauf das Kürzel der Zeitschrift, gefolgt von der Bandangabe (mit oder ohne vorangestelltem „Bd.“), dem Erscheinungsjahr in Klammern sowie einem weiteren Komma und zuletzt der Anfangs- und/oder Zitatseite (mit oder ohne vorangestelltem „S.“).

83 Mangels fertiger Softwarelösungen waren dafür eigene Algorithmen zu entwickeln, die in PHP umgesetzt wurden.

84 Ausnahmen bestätigen die Regel: FA bspw. wird sowohl für „Fachanwalt Arbeitsrecht“ verwendet als auch für „FinanzArchiv“. Solche Unklarheiten sind aber kein Problem speziell der Zitationsanalyse. 
- Entscheidungssammlungen werden so zitiert, dass auf das Kürzel der Entscheidungssammlung die Bandnummer folgt, darauf ein Komma sowie die Anfangsund/oder Zitatseite.

- Fundstellen in allen anderen Zeitschriften bestehen aus dem Autor des Textes, oft gefolgt von einem Komma, dem Zeitschriftenkürzel sowie Erscheinungsjahr, einem weiteren Komma und der Anfangs- und Zitatseite (wiederum mit oder ohne vorangestelltem „S.“). 85

Diese Konventionen gelten für die Zitierung fast aller deutschen Rechtszeitschriften, mit Ausnahme weniger Entscheidungssammlungen (z.B. LM und AP) und Zeitschriften (z.B. NWB und WuB). Daher war es möglich, die vorliegenden Fußnoten automatisiert nach Fundstellen zu rastern, indem jedes Zeitschriftenkürzel festgestellt und aus den nachfolgenden Buchstaben, Satzzeichen und Zahlen automatisiert diejenigen Fundstellen rekonstruiert wurden, die einer der drei Konventionen entsprechen. ${ }^{86}$ Dies ergab insgesamt 1.623.491 Zitate (Erwähnungen) von 509.353 unterschiedlichen Fundstellen. Diese Fundstellen lagen überwiegend im dritten Format vor, mit Ausnahme von 12.210 Fundstellen im ersten und $30.382 \mathrm{im}$ zweiten $\mathrm{Zi}$ tierformat. Insgesamt wurden dabei 874 der 1.371 gelisteten Zeitschriftenkürzel verwendet.

Im nächsten Schritt wurde für jede Fundstelle der Name des jeweiligen Autors ermittelt. Dazu wurde für alle Vorkommen einer bestimmten Fundstelle das ihr am häufigsten vorangehende Wort errechnet, das mindestens einen Großbuchstaben enthielt. ${ }^{87}$ Dieses Wort wurde als Autorenname behandelt, was bei einer stichprobenartigen Kontrolle recht zuverlässig zu funktionieren schien. Auf diese Art konnte 1.367.295 Zitaten (84,2 \%) ein Autor zugeordnet werden.

Schließlich wurden Zitate ausgeschlossen, die auf ein „=“ folgten. Dabei handelt es sich erfahrungsgemäß (und nach Stichprobenkontrolle) um parallele Veröffentlichungen desselben Textes - zumeist Gerichtsentscheidungen -, die oft nur der Vollständigkeit halber zitiert werden und für die Zitationsanalyse ausgeklammert werden sollten, um die Bedeutung des jeweiligen Textes nicht zu überschätzen. In diesem Schritt fielen 300.341 Zitate von 103.549 Fundstellen weg.

In einigen Fällen erzeugte die bisher dargestellte Aufbereitung offenkundig unsinnige Fundstellen, dann wurde jeweils stichprobenartig von Hand nachgeprüft, ob der Fehler auf der Auswertungsroutine oder auf Schreib- oder Zitierfehlern im ausge-

85 Soweit Aufsätze ohne Anfangsseite (also nur mit der Zitatseite) zitiert wurden, schien es sich um sporadische Abweichungen und nicht um eine eigenständige Zitierkonvention zu handeln.

86 Dabei wurden sog. reguläre Suchausdrücke verwendet, die ich auf Nachfrage gern mitteile. Eine gesonderte Korrekturroutine war erforderlich für Zeitschriftenabkürzungen, die zugleich Worte im Text darstellen konnten (z.B. „Staat“, „Konzern“, „Verwaltung“, etc.).

87 Dieses Wort durfte auch Schrägstriche enthalten, um Mehrautorenschaften einzufangen. 
werteten Urtext beruhte. Nach schrittweiser Verbesserung der Auswertungsroutine verblieben schließlich nur solche Fehler, die auch ein verständiger Leser nicht ohne Weiteres hätte korrigieren können. Sie erforderten eine drastische Korrektur durch Ausschluss der von ihnen betroffenen Datensätze. Ausgeschlossen wurden in diesem Schritt

- 141 Fundstellen mit einem vermeintlichen Erscheinungsjahr nach 2012, dem Jahr der aktuellsten ausgewerteten Aufsätze.

- 423 Fundstellen mit einem vermeintlichen Erscheinungsjahr vor 1818, dem Gründungsjahr der wohl ältesten noch bestehenden Zeitschrift „Archiv für die civilistische Praxis" (AcP). 88

- 30 Fundstellen mit einem vermeintlichen Erscheinungsjahr nach dem des zitierenden Textes.

Schließlich wurde der Datenbestand in einem letzten Schritt so gekürzt, dass je untersuchtem Aufsatz jede Fundstelle nur einmal gezählt wurde, auch wenn sie im selben Text noch mehrmals auftauchte. Das sollte Verzerrungen ausschließen, die dadurch entstehen, dass Folgezitate nicht immer ausgeschrieben, sondern oft mit „a.a.O.“ oder vergleichbaren Angaben abgekürzt werden; zudem stellt es einen Gleichlauf zur Zitationsanalyse in den USA her, die Zitate durchweg nur einmal auf Artikelebene zählt. Damit verblieben zuletzt 895.091 auswertbare Zitate von 446.561 Fundstellen.

\section{Datenauswertung}

Von den vielen denkbaren Forschungsfragen wurden vorerst vier eher willkürlich herausgegriffen: 1. Welcher Anteil juristischer Veröffentlichungen wird überhaupt zitiert? 2. Welche Zeitschriften werden am häufigsten zitiert? 3. Welche Zeitschriftenartikel haben den größten Einfluss? 4. Wie lassen sich „Klassiker“ im Schrifttum identifizieren?

\section{Publikationsflut und dunkle Materie}

In allen Wissenschaften wird gelegentlich die zunehmende Publikationsflut beklagt, die dazu führt, dass viele Arbeiten gar nicht mehr zur Kenntnis genommen, geschweige denn produktiv verarbeitet werden können. Selbst wenn man nur die renommiertesten Wissenschaftszeitschriften betrachtet und Selbstzitate mitzählt, werden wenig mehr als $40 \%$ der Zeitschriftenbeiträge in den ersten fünf Jahren nach ihrem Erscheinen überhaupt zitiert. ${ }^{89}$ In den Sozialwissenschaften bleiben schät-

88 Die hernach älteste Fundstelle war Nahmer, AcP 11 (1828), S. 298, zit. in Sangmeister, BB 1992, 323 Fn. 12.

89 S. W. Trimble/W. W. Grody/B. McKelvey/M. Gad-el-Hak, The Glut of Academic Publishing: A Call for a New Culture, 23 Acad. Quest. 2010, S. 276 (277) m.w.N. 
zungsweise $48 \%$ der Monographien ${ }^{90}$ und 74,7 \% der Zeitschriftenbeiträge ${ }^{91}$ in den vier Jahren nach ihrem Erscheinen unzitiert. Für die US-amerikanische Rechtswissenschaft wurde geschätzt, dass von allen Zeitschriftenbeiträgen zwischen 1981 und 1993 ganze $57 \%$ bis 1997 kein einziges Mal zitiert wurden. ${ }^{92}$ Nun heißt das natürlich nicht, dass diese Arbeiten nicht trotzdem gelesen werden und den Diskurs beeinflussen - einige empirische Schätzungen gehen davon aus, dass mitunter $70 \%$ der Literatur, auf die ein wissenschaftlicher Text zurückgreift, unzitiert bleiben. ${ }^{93}$ Andererseits werden viele Arbeiten zitiert, obwohl sie nicht gelesen wurden - auch hier belaufen sich empirische Schätzungen auf $70 \%$ und mehr. ${ }^{94}$ Beide Schätzungen betreffen andere Fachgebiete als die Rechtswissenschaft, auch wenn die zugrundeliegenden Phänomene Juristen ebenso vertraut sein dürften. ${ }^{95} \mathrm{Ob}$ Juristen häufiger ungelesene Texte zitieren oder gelesene Texte nicht zitieren, ist unklar. Daher lässt sich der Anteil der nie zitierten Arbeiten nur als erste Näherung für jenen Teil des Diskurses behandeln, der hier mit einer Anleihe aus der Physik als „dunkle Materie“ bezeichnet wird. ${ }^{96}$

Um diesen Anteil zu ermitteln, bedurfte es neben den bereits erhobenen Daten auch Angaben darüber, wieviele zitierbare Arbeiten überhaupt existieren. Mangels offizieller Statistiken wurde diese Gesamtmenge behelfsweise anhand der juris-Datenbank abgeschätzt: Für jede der 874 zitierten Zeitschriften wurde - soweit möglich - die Anzahl der juris-Suchtreffer (Rechtsprechung bzw. Literaturnachweise) für den Zeitraum 2000 bis 2010 ermittelt. ${ }^{97}$ Diese wurden anschließend mit der Anzahl der in den aufbereiteten Zeitschriften vorgefundenen Fundstellen im gleichen Zeitraum verglichen. Dadurch lässt sich ermitteln, wieviele der von juris indizierten Fundstellen niemals in den vorliegend untersuchten Zeitschriften aufgetaucht sind. Dabei konnten freilich nur Fundstellen einbezogen werden, die mit Jahresangabe zitiert wurden,

90 R. Tang, Citation Characteristics and Intellectual Acceptance of Scholarly Monographs, 69 Coll. \& Res. Lib. 2008, S. 356 (361) für Zitate seit Erscheinen bzgl. 375 zufällig gewählter Sachbücher vor 2004, je anteilig aus Geschichte, Psychologie und Ökonomik.

91 D. P. Hamilton, Research Papers: Who's Uncited Now?, 251 Science 1991, S. 25.

92 D. L. Rhode, Legal Scholarship, 115 Harv. L. Rev. 2002, S. 1327 (1331 Fn. 16).

93 So MacRoberts/MacRoberts, Problems (Fn. 60), S. 436 für die Fachgebiete Botanik, Zoologie, Ethologie, Soziologie und Psychologie; neuere Nachw. bei M. H. MacRoberts/B. R. MacRoberts, Problems of Citation Analysis: A Study of Uncited and Seldom-Cited Influences, 61 J. Am. Soc. Inf. Sci. Tech. 2010, S. 1.

94 S.o. Fn. 56.

95 Auch in der Rechtswissenschaft sind „Fußnoten-Phantome“ (Begriff von C. Grube, Der Bluff mit dem Fußnoten-Phantom, Süddt. Ztg. 24.11.2011, http://sz.de/1.1217376) und Blindzitate berüchtigt, vgl. nur VG Osnabrück, Urt. v. 23.11.2009, Az. 9 A 5/09, juris Rn. 50.

96 Dunkle Materie ist solche, die sich zwar durch Wechselwirkungen mit sichtbarer Materie (Schwer$\mathrm{kraft}$ ) bemerkbar macht, aber nie direkt beobachtet wurde - also ein rein theoretisches Konstrukt. Ich meine, mich zu erinnern, dass H.-B. Schäfer diese Metapher in einem Vortrag über die europäische Rechtsökonomik benutzte.

97 Die Beschränkung des Zeitraums verringert das Risiko, dass einzelne Zeitschriften in juris nicht weit genug zurückreichen, oder dass einzelne Hefte noch nicht indiziert sind. Die Erhebung erfolgte am 15.2.2014. 
sowie Zeitschriften, die juris zumindest rudimentär indiziert. ${ }^{98}$ Zitationsraten wurden konservativ gekappt, wo mehr Fundstellen zitiert waren als juris verzeichnet.

Beispiel: Für die ZGR wurden 670 verschiedene Fundstellen zwischen 2000 und 2010 zitiert, obwohl juris in diesem Zeitraum nur 340 Suchtreffer verzeichnet. Folglich gelten $100 \%$ der ZGR-Inhalte als tatsächlich zitiert.

Diese Auswertung ergab, dass insgesamt - also über alle in juris indizierten Zeitschriften hinweg - 92-95\% der Rechtsprechungs- und 69-77\% der Aufsatzfundstellen 2000-2010 in keiner der hier ausgewerteten Zeitschriften bis 2012 zitiert worden waren. ${ }^{99}$ Allerdings gehen in diese Schätzung auch zahlreiche Zeitschriften ein, die schon thematisch nicht in Betracht kommen, durch die vorliegenden Zeitschriften zitiert zu werden. Um die Schätzung auch gegen solche Verzerrungen abzusichern, wurde die Auswertung auf die vorliegend ausgewerteten Zeitschriften beschränkt:

\begin{tabular}{|c|c|c|c|c|c|c|c|}
\hline \multirow[b]{2}{*}{ Zeitschrift } & \multicolumn{2}{|c|}{ Juris-Treffer } & \multicolumn{3}{|c|}{ Zitierte Fundstellen } & \multicolumn{2}{|c|}{ Nichtzit. / juris-Treffer } \\
\hline & Aufsätze & Rechtspr. & Aufsätze & Rechtspr. & unklar $^{100}$ & Aufsätze & Rechtspr. \\
\hline AG & 638 & 1.499 & 1.052 & 386 & 521 & $0 \%$ & $40-74 \%$ \\
\hline BB & 4.375 & 10.273 & 4.072 & 523 & 2.423 & $0-7 \%$ & $71-95 \%$ \\
\hline DZWIR & 728 & 1.449 & 316 & 157 & 467 & $0-57 \%$ & $57-89 \%$ \\
\hline JA & 1.707 & 1.183 & 489 & 16 & 27 & $70-71 \%$ & $96-99 \%$ \\
\hline $\mathrm{JuS}$ & 2.361 & 2.003 & 1.384 & 28 & 68 & $39-41 \%$ & $95-99 \%$ \\
\hline MDR & 1.207 & 14.373 & 621 & 389 & 1.902 & $0-49 \%$ & $84-97 \%$ \\
\hline NJW & 5.122 & 28.611 & 3.551 & 6.914 & 1.150 & $8-31 \%$ & $72-76 \%$ \\
\hline NStZ & 875 & 7.837 & 610 & 899 & 365 & $0-30 \%$ & $84-89 \%$ \\
\hline NVwZ & 1.736 & 11.357 & 1.222 & 1.613 & 197 & $18-30 \%$ & $84-86 \%$ \\
\hline NZG & 1.586 & 3.511 & 1.506 & 1.611 & 189 & $0-5 \%$ & $49-54 \%$ \\
\hline NZM & 824 & 5.134 & 794 & 1.954 & 352 & $0-4 \%$ & $55-62 \%$ \\
\hline ZGR & 324 & 16 & 650 & 7 & 13 & $0 \%$ & $0-56 \%$ \\
\hline ZIP & 1.474 & 6.846 & 1.980 & 2.515 & 541 & $0 \%$ & $55-63 \%$ \\
\hline ZRP & 1.232 & 0 & 724 & 4 & 34 & $39-41 \%$ & $0 \%$ \\
\hline Insgesamt & 24.189 & 94.092 & 18.971 & 17.016 & 8.249 & $0-22 \%$ & $73-82 \%$ \\
\hline
\end{tabular}

Tab. 4: Dunkle Materie in jeder der vorliegend ausgewerteten Zeitschriften, errechnet als Anteil der nicht zitierten Fundstellen an allen juris-Treffern im Zeitraum 2000-2010, jeweils getrennt nach Aufsätzen und Rechtsprechung

98 Als nicht wenigstens rudimentär indiziert wurden Zeitschriften behandelt, die insgesamt weniger als 44 Suchtreffer verzeichneten (= 1 pro Quartal). Das betraf 192 Zeitschriften.

99 Fürdas obere Ende der jeweiligen Spanne wurden Zitate ignoriert, beidenennicht klar war, ob sie sich auf Rechtsprechung oder Aufsätze beziehen (vgl. nächste Fn.), für das untere Ende wurden diese Zitate in beiden Kategorien gezählt.

100 Für die Fundstellen in dieser Spalte ließ sich kein Autor extrahieren. Nach stichprobenartiger Kontrolle scheinen die meisten davon Rechtsprechung zu beinhalten, deshalb ist von den rechts angegebenen Prozentspannen für Aufsätze eher das obere Ende, für Rechtsprechung eher das untere Ende realistisch. 
Damit ist festzustellen, dass bei vorsichtiger Schätzung ungefähr vier Fünftel der Rechtsprechung und ein Fünftel der Aufsätze nicht in der Zeitschriftenliteratur zitiert werden. Das ist für die Rechtsprechung insofern nicht überraschend, als Entscheidungen meist in vielen Zeitschriften abgedruckt, aber immer aus denselben Zeitschriften zitiert werden. Bemerkenswert ist dagegen bei den Aufsätzen die Verteilung „dunkler Materie“: Mehrere Zeitschriften (darunter die Archivzeitschrift ZGR) enthalten gar keine, während die Ausbildungszeitschrift JA selbst bei konservativer Schätzung zu mehr als zwei Dritteln daraus besteht. Selbst unter Berücksichtigung dessen weist die deutsche Rechtswissenschaft allerdings relativ wenig „dunkle Materie" auf, wenn man sie mit anderen Disziplinen vergleicht.

\section{Zeitschriftenranking}

Ein wichtiger Anwendungsbereich der Zitationsanalyse ist das sog. Zeitschriftenranking, also die Untersuchung, welche Zeitschriften am häufigsten zitiert werden. Obwohl solche Rankings international weit verbreitet sind, ${ }^{101}$ existiert für die deutsche Rechtswissenschaft bislang nur ein einziger Modellversuch, für den Rechtswissenschaftler per E-Mail nach den „qualitativ bedeutendsten“ Zeitschriften ihres Fachgebiets sowie des fachgebietsübergreifenden Diskurses befragt wurden. ${ }^{102}$ Die Zitationsanalyse ermöglicht es, solche subjektiven Wahrnehmungen am tatsächlichen Einfluss einzelner Zeitschriften zu spiegeln.

Betrachtet man zunächst für jede untersuchte Zeitschrift, auf welche Zeitschriften die meisten ihrer Zitate verweisen, ergibt sich, dass zwei Drittel der Zeitschriften (AG, BB, MDR, NJW, NStZ, NVwZ, NZG, NZM und ZIP) am häufigsten ihre eigenen Texte zitieren (Anteil der Selbstzitate insgesamt: 20,7 \%). Solche Selbstzitate verzerren die Auswertung, können aber auch nicht einfach entfernt werden, weil damit der Einfluss der betreffenden Zeitschriften wiederum stark unterschätzt würde. Deshalb kam der folgende Korrekturmechanismus zur Anwendung: Für jede Zeitschrift wurden Eigenzitate so oft berücksichtigt, als hätte sich die Zeitschrift mit derselben Häufigkeit selbst zitiert wie sie von anderen Zeitschrift desselben Fachgebiets durchschnittlich zitiert wurde.

Beispiel: Wenn in AG, DZWIR, NZG, ZGR und ZIP $10 \%$ der Zitate auf BB-Artikel verweisen, im BB aber 25,7\% der Zitate, wurden knapp zwei Drittel dieser Zitate (genauer: $61,1 \%=1-10 / 25,7)$ ignoriert.

Dieses Verfahren ist natürlich nur möglich, wo mehrere Zeitschriften des selben Fachgebiets vorliegen, und versagt auch für separate, aber eng geführte Beiveröffentlichungen wie die „Rechtsprechungs-Reports“ einiger Zeitschriften, deren Ein-

101 Für die US-Rechtswissenschaft v.a. Shapiro, Law Reviews (Fn. 18) sowie das "Law Journal Rankings Project” der Washington \& Lee University (Law Library) unter http://lawlib.wlu.edu/LJ; für Europa einschränkend Van Gestel/Vranken, Research (Fn. 39), S. 921: "most Western European countries do not have a ranking of law journals and citation indexes yet.".

102 Gröls/Gröls, Ranking (Fn. 35), 34. 
fluss deshalb stark überschätzt werden dürfte. Deshalb wurde probehalber eine Rangliste für die beiden durch mehrere Zeitschriften vertretenen Rechtsgebiete erstellt und unter Ausschluss von Rechtsprechungszitaten wiederholt:

\begin{tabular}{|c|c|c|c|c|c|c|c|c|}
\hline \multirow{2}{*}{ Rang } & \multicolumn{2}{|c|}{ Allg. Zeitschriften } & \multicolumn{2}{c|}{ Allg. Zs. ohne Rspr. } & \multicolumn{2}{c|}{ Wirtschaftsrecht-Zs. } & \multicolumn{2}{c|}{ WirtR-Zs. ohne Rspr. } \\
& $\begin{array}{c}\text { Abkür- } \\
\text { zung }\end{array}$ & $\begin{array}{c}\text { Zitat- } \\
\text { Anteil }\end{array}$ & $\begin{array}{c}\text { Abkür- } \\
\text { zung }\end{array}$ & $\begin{array}{c}\text { Zitat- } \\
\text { Anteil }\end{array}$ & $\begin{array}{c}\text { Abkür- } \\
\text { zung }\end{array}$ & $\begin{array}{c}\text { Zitat- } \\
\text { Anteil }\end{array}$ & $\begin{array}{c}\text { Abkür- } \\
\text { zung }\end{array}$ & $\begin{array}{c}\text { Zitat- } \\
\text { Anteil }\end{array}$ \\
\hline 1 & NJW & $8,0 \%$ & NJW & $5,8 \%$ & NJW & $8,6 \%$ & DB & $9,9 \%$ \\
\hline 2 & BVerfGE & $6,5 \%$ & JZ & $5,5 \%$ & DB & $7,6 \%$ & ZIP & $7,2 \%$ \\
\hline 3 & NJW-RR & $5,9 \%$ & BB & $3,8 \%$ & BGHZ & $7,5 \%$ & BB & $5,7 \%$ \\
\hline 4 & BGHZ & $5,9 \%$ & JuS & $3,0 \%$ & ZIP & $6,5 \%$ & NJW & $5,3 \%$ \\
\hline 5 & FamRZ & $3,8 \%$ & FamRZ & $2,6 \%$ & WM & $4,6 \%$ & DStR/AG & $5,1 \%$ \\
\hline
\end{tabular}

Tab. 5: Meistzitierte Zeitschriften im allgemeinen und wirtschaftsrechtlichen Schrifttum, jeweils mit ihrem Anteil an allen Zitaten insgesamt (mit bzw. ohne Rechtsprechung)

Aus der Tabelle ergibt sich bereits, dass die NJW sowie die amtliche Entscheidungssammlung des BGH in Zivilsachen sowohl in allgemeinen als auch in wirtschaftsrechtlichen Zeitschriften besonders häufig zitiert werden. Schließt man allerdings Rechtsprechungszitate aus, verändert sich die Reihung und die NJW fällt im Wirtschaftsrecht zurück, während von unten Zeitschriften aufrücken, die zuvor durch die Einbeziehung von Rechtsprechung benachteiligt worden waren. Das gilt im allgemeinen Schrifttum vor allem für die JZ sowie die JuS, deren Rechtsprechungsteil bekanntlich kaum zitiert wird (oben Tab. 4). In der weiteren Auswertung wurden Rechtsprechungszitate deshalb ausgeblendet.

Eine weitere Verzerrung entsteht in der bisherigen Auswertung dadurch, dass manche Zeitschriften schlicht öfter erscheinen als andere, folglich mehr Beiträge versammeln und schon deshalb häufiger zitiert werden sollten. ${ }^{103}$ Deshalb gibt die obige Tabelle zwar die Sichtbarkeit der einzelnen Zeitschriften wieder, doch ein besserer Gradmesser für deren Einfluss ergibt sich, wenn man die tatsächliche Häufigkeit, mit der jede Zeitschrift zitiert wird, ins Verhältnis zur Anzahl der darin veröffentlichten Texte setzt - abgeschätzt wiederum anhand der Trefferanzahl in juris. ${ }^{104}$ Daraus ergibt sich, welche Zeitschriften öfter zitiert werden als ihr rein mengenmäßiger Anteil an den Veröffentlichungen erwarten lassen würde.

103 Gleiches gilt genaugenommen für Zeitschriften mit längeren Beiträgen, vgl. Landes/Posner, Articles (Fn. 26), S. 827: "One expects that, other things being equal, a longer article [...] will be cited more frequently.".

104 S.o. bei Fn. 97; berücksichtigt wurden erneut nur mindestens rudimentär indizierte Zeitschriften (Fn. 98). Mathematisch wurde also die jeweilige \{Anzahl der Zitate auf die konkrete Zeitschrift\} mit der \{Gesamtzahl der juris-Suchtreffer 2000-2010\} multipliziert und durch die \{Gesamtzahl der Zitate\} sowie die \{Anzahl der juris-Suchtreffer 2000-2010 für die konkrete Zeitschrift\} dividiert. 
Beispiel: Auf die „JuristenZeitung“ (JZ) entfallen 4,3\% aller Zitate der Jahre 2000-2010 aus den fünf allgemeinen Zeitschriften (ohne Rechtsprechung). Zugleich entfallen auf die JZ aber nur 0,27\% aller juris-Suchtreffer im gleichen Zeitraum. Damit erzielt die JZ einen gut 15 Mal höheren Einfluss als die bloße Anzahl ihrer Beiträge erklärt.

Damit ergeben sich folgende Rangfolgen der Zeitschriften, deren diskursiver Einfluss ihre reale Materialmenge am weitesten überragt: ${ }^{105}$

\begin{tabular}{|c|c|c|c|c|c|c|c|}
\hline \multicolumn{2}{|c|}{ Wirtschaftsrecht } & \multicolumn{2}{|r|}{ Strafrecht ${ }^{106}$} & \multicolumn{2}{|c|}{ Verwaltungsrecht ${ }^{107}$} & \multicolumn{2}{|r|}{ Allgemein } \\
\hline 1 & $Z_{\text {GR }}^{(2)}$ & 1 & $\mathrm{NStZ}_{(1)}$ & 1 & $\mathrm{NVwZ}_{(3)}$ & 1 & $\mathrm{RRa}$ \\
\hline 2 & $\mathrm{ZHR}_{(1)}$ & 2 & StraFo $_{(5)}$ & 2 & UPR & 2 & $\mathrm{JZ}_{(1)}$ \\
\hline 3 & $\mathrm{AG}_{(4)}$ & 3 & $\mathrm{ZIS}_{(7)}$ & 3 & Die Verw. ${ }_{(6)}$ & 3 & RDV \\
\hline 4 & $\mathrm{ZIP}_{(3)}$ & 4 & HRRS & 4 & DVBl. $_{(4)}$ & 4 & ZIP \\
\hline 5 & $\mathrm{NZG}_{(6)}$ & 5 & $\mathrm{StV}_{(3)}$ & 5 & $\mathrm{NuR}$ & 5 & $\mathrm{AcP}_{(3)}$ \\
\hline 6 & $\mathrm{WM}_{(7)}$ & 6 & $\mathrm{ZStW}_{(4)}$ & 6 & NZBau & 6 & ZGS \\
\hline 7 & GWR & 7 & $\mathrm{GA}_{(2)}$ & 7 & ZUR & 7 & ZKM \\
\hline 8 & Der Konzern & 8 & ZJJ & 8 & VerwArch $_{(7)}$ & 8 & Die Verw. \\
\hline 9 & BFuP & 9 & $\mathrm{ZfL}$ & 9 & DÖV $_{(5)}$ & 9 & NStZ \\
\hline 10 & DB & 10 & KritV & 10 & AbfallR & 10 & AöR $_{(4)}$ \\
\hline 11 & ZBB & 11 & MschrKrim & 11 & ZBR & 11 & NZM \\
\hline 12 & $\mathrm{GmbHR}_{(5)}$ & 12 & JR & 12 & $\mathrm{ZfW}$ & 12 & ZGR \\
\hline 13 & BKR & 13 & KrimPäd & 13 & ZG & 13 & NZBau \\
\hline 14 & WPg & 14 & $\mathrm{JZ}$ & 14 & $\mathrm{AöR}_{(1)}$ & 14 & $\mathrm{ZRP}_{(14)}$ \\
\hline 15 & NZI & 15 & NK & 15 & EurUP & 15 & EuGRZ \\
\hline
\end{tabular}

Tab. 6: Überragend einflussreiche Zeitschriften nach Rechtsgebiet, mit kleingedruckten Rangpositionen (soweit vorhanden) nach Gröls/Gröls, Ranking (Fn. 35)

Zum Vergleich mit der früheren umfragebasierten Rangliste (dazu oben bei Fn. 102) enthält die Tabelle deren Rangpositionen in Klammern. Dabei fallen sowohl klare Übereinstimmungen als auch überraschende Abweichungen ins Auge: Im Wirtschaftsrecht sind die ersten sechs Ränge nahezu identisch belegt, und auch im Strafrecht liegen mit einer Ausnahme die selben Zeitschriften auf den ersten sieben Rängen. Geringer ist die Übereinstimmung im Verwaltungsrecht, und im allgemeinen Schrifttum fehlt sie fast völlig. Letzteres könnte daran liegen, dass die im früheren Ranking gestellte Frage nach „Rechtszeitschriften, die über ein einzelnes Rechtsgebiet hinaus Bedeutung für alle Felder entfalten " 108 weiten Interpretationsspielraum lässt und nicht unbedingt mit dem hier verwendeten Kriterium der „Allgemeinheit“

105 Anders gewendet geben die Rangfolgen an, welche Zeitschriften im jeweiligen Rechtsgebiet den geringsten Anteil dunkler Materie aufweisen (dazu oben D. III.1.), denn darauf läuft die in der vorigen Fn. angegebene Formel hinaus, wenn man deren Ergebnisse ordinal skaliert.

106 Da hier nur die NStZ ausgewertet werden konnte, war eine Herausrechnung von Selbstzitaten nicht möglich.

107 Da hier nur die NVwZ ausgewertet werden konnte, war eine Herausrechnung von Selbstzitaten nicht möglich.

108 Gröls/Gröls, Ranking (Fn. 35), S. 35. 
zusammenfällt. Zu den Überraschungen in der hiesigen Rangliste gehören wohl vor allem im Wirtschaftsrecht die noch junge GWR (gegr. 2009), im Strafrecht die HRRS als reine Online-Zeitschrift und im allgemeinen Schrifttum Zeitschriften wie RRa und RDV. Weniger überraschend ist dagegen vielleicht die hohe Platzierung der ZGS, deren Einfluss im Zeitraum 2000-2010 maßgeblich auf der Schuldrechtsmodernisierung beruhen dürfte. ${ }^{109}$

Diese wenigen Beobachtungen mögen genügen, um zu belegen, dass die Zitationsanalyse einerseits sehr gut mit Expertenurteilen korreliert, andererseits aber auch wertvolle Beiträge zu deren Ergänzung und Überprüfung leisten kann.

\section{Einflussmessung einzelner Artikel}

Auch wenn das Zeitschriftenranking eine der wichtigsten Anwendungen der Zitationsanalyse in den USA geworden ist, konzentrierte sich die juristische Zitationsanalyse ursprünglich ${ }^{110}$ auf die Frage, welche Primärtexte am häufigsten zitiert werden. Das ist auch deshalb sinnvoll, weil der Einfluss einer Zeitschrift keinen zwingenden Rückschluss auf den Einfluss ihrer einzelnen Beiträge erlaubt. ${ }^{111}$

Die vorliegende Untersuchung konnte erstmals auch für die deutsche Rechtswissenschaft den Einfluss einzelner Artikel erheben. Über alle 34.334 hier untersuchten Artikel hinweg ergab sich folgende Rangliste der zehn meistzitierten Zeitschriftenfundstellen:

109 Allg. zu Zitierungen der ZGS Siems, Wie häufig (Fn. 7), S. 90.

110 Und zwar bei ihrer Erfindung ebenso wie bei der Wiederentdeckung durch Shapiro, s.o. bei Fn. 15.

111 Van Gestel/Vranken, Research (Fn. 39), S. 918; empirisch Brophy, Signaling (Fn. 43), S. 238: "some articles in the less elite journals are more heavily cited than many articles in even the most elite journal”; für andere Disziplinen ausf. B. Brembs/K. Button/M. Munafò, Deep impact: unintended consequences of journal rank, Frontiers Hum. Neurosc. 2013, S. 1 (6). 


\begin{tabular}{|c|l|l|c|c|}
\hline Rang & Fundstelle & Titel & Artikel & Anteil \\
\hline 1 & BVerfGE 65, 1 & $\begin{array}{l}\text { Urt. v. 15.12.1983, Az. 1 BvR 209/83 u.a. (Volkszäh- } \\
\text { lung) }\end{array}$ & 450 & $1,31 \%$ \\
\hline 2 & BVerfGE 50, 290 & Urt. v. 1.3.1979, Az. 1 BvR 532/77 u.a. (MitbestG) & 309 & $0,90 \%$ \\
\hline 3 & BVerfGE 7, 198 & Urt. v. 15.1.1958, Az. 1 BvR 400/51 (Lüth) & 297 & $0,86 \%$ \\
\hline 4 & BGHZ 83, 122 & Urt. v. 25.2.1982, Az. II ZR 174/80 (Holzmüller) & 290 & $0,84 \%$ \\
\hline 5 & BVerfGE 49, 89 & Beschl. v. 8.8.1978, Az. 2 BvL 8/77 (Schneller Brüter) & 283 & $0,82 \%$ \\
\hline 6 & BGHZ 135, 244 & $\begin{array}{l}\text { Urt. v. 21.4.1997, Az. II ZR 175/95 (ARAG/Garmen- } \\
\text { beck) }\end{array}$ & 276 & $0,80 \%$ \\
\hline 7 & BVerfGE 58, 300 & $\begin{array}{l}\text { Beschl. v. 15.7.1981, Az. 1 BvL 77/78 (Nassauskie- } \\
\text { sung) }\end{array}$ & 212 & $0,62 \%$ \\
\hline 8 & BVerfGE 53, 30 & $\begin{array}{l}\text { Beschl. v. 20.12.1979, Az. 1 BvR 385/77 (Mülheim- } \\
\text { Kälich) }\end{array}$ & 210 & $0,61 \%$ \\
\hline 9 & BVerfGE 39, 1 & $\begin{array}{l}\text { Urt. v. 25.2.1975, Az. 1 BvF 1/74 (Schwangerschafts- } \\
\text { abbruch) }\end{array}$ & 204 & $0,59 \%$ \\
\hline 10 & BVerfGE 7, 377 & $\begin{array}{l}\text { Urt. v. 11.6.1958, Az. 1 BvR 596/56 (Apothekenur- } \\
\text { teil) }\end{array}$ & 203 & $0,59 \%$ \\
\hline 10 & BGHZ 146, 341 & $\begin{array}{l}\text { Urt. v. 29.1.2001, Az. II ZR 331/00 (GbR: Rechtsfä- } \\
\text { higkeit) }\end{array}$ & 203 & $0,59 \%$ \\
\hline
\end{tabular}

Tab. 7: Meistzitierte Fundstellen in den untersuchten Zeitschriften, jeweils mit Anzabl der zitierenden Artikel und Anteil an allen Artikeln

Das Volkszählungsurteil des Bundesverfassungsgerichts ist in den hier untersuchten Zeitschriften also nicht nur das meistzitierte Urteil, sondern die meistzitierte Fundstelle überhaupt - die einzige, die mindestens in jedem Hundertsten der untersuchten Artikel auftaucht. Beim Blick auf die weiteren Platzierungen sind zwei Tatsachen unmittelbar augenfällig:

Erstens entstammen die zehn meistzitierten Fundstellen durchweg der Rechtsprechung. Dies gilt sogar noch für 64 weitere Rangpositionen; erst auf Rang 75 kommt ein Aufsatz aus der Wissenschaft, der mit 81 Zitationen kaum ein Fünftel so oft herangezogen wurde wie die meistzitierte Rechtsprechung. Das ist im internationalen Vergleich scheinbar ungewöhnlich, weil dort Aufsätze mit Tausenden Zitationen die Ranglisten anführen, ${ }^{112}$ lässt sich aber zwanglos schon daraus erklären, dass dort teilweise Vollerhebungen durchgeführt werden, ${ }^{113}$ während hier nur eine relativ kleine Stichprobe untersucht werden konnte. Vor diesem Hintergrund liegt die hier festgestellte Zitationshäufigkeit durchaus in einer vergleichbaren Größenordnung, ${ }^{114}$ denn trotz der viel größeren Stichproben werden auch in den USA die wenigsten Zeitschriftenbeiträge überhaupt $200 \mathrm{Mal}$ zitiert:

112 Vgl. Shapiro/Pearse, [Tabellenwerk zu All Time (Fn. 17)], Mich. L. Rev. First Impressions 2012 (http://www.michiganlawreview.org/assets/110/8/shapiropearse_supplementaltables.pdf).

113 Shapiro/Pearse, All Time (Fn. 17), S. 1486 erheben Zitationsdaten für 1,4 Millionen Aufsätze.

114 Teilt man die Zitationshäufigkeit der meistzitierten juristischen Aufsätze in den USA durch die Stichprobengröße der Erhebung (vorige Fn.), entstehen Anteile von 0,37 \% bzw. 0,26 \%, die den hiesigen sehr nahekommen. 
"it is surprising that so few articles manage to accrete 204 citations (the cutoff number in the top one hundred list). The implication is that law is a relatively decentralized and competitive field of scholarship, as distinct from one dominated by a handful of scholars to whom the rest defer." 115

Zweitens fällt auf, dass die zehn meistzitierten Gerichtsentscheidungen deutlich von wirtschaftsrechtlichen Fragen dominiert sind, was zweifellos dem großen Gewicht wirtschaftsrechtlicher Zeitschriften im vorliegend untersuchten Zeitschriftenbestand geschuldet ist. Da für eine nähere Untersuchung der wirtschaftsrechtlichen Zitationsmuster vorliegend kein Raum ist, wird die Auswertung deshalb im Weiteren auf die fünf allgemeinen Zeitschriften (JA, JuS, MDR, NJW, ZRP) mit ihren insgesamt 14.694 Artikeln beschränkt. Für diese ergibt sich folgende Rangliste:

\begin{tabular}{|c|c|c|c|c|}
\hline Rang & Fundstelle & Titel & Artikel & Anteil \\
\hline 1 & BVerfGE 65,1 & $\begin{array}{l}\text { Urt. v. 15.12.1983, Az. } 1 \text { BvR 209/83 u.a. (Volks- } \\
\text { zählung) }\end{array}$ & 246 & $0,72 \%$ \\
\hline 2 & BVerfGE 7, 198 & Urt. v. 15.1.1958, Az. 1 BvR 400/51 (Lüth) & 217 & $0,63 \%$ \\
\hline 3 & BVerfGE 49,89 & $\begin{array}{l}\text { Beschl. v. 8.8.1978, Az. } 2 \text { BvL 8/77 (Schneller Brü- } \\
\text { ter) }\end{array}$ & 141 & $0,41 \%$ \\
\hline 4 & BVerfGE 39,1 & $\begin{array}{l}\text { Urt. v. 25.2.1975, Az. } 1 \text { BvF 1/74 (Schwanger- } \\
\text { schaftsabbruch) }\end{array}$ & 138 & $0,40 \%$ \\
\hline 5 & BVerfGE 50, 290 & Urt. v. 1.3.1979, Az. 1 BvR 532/77 u.a. (MitbestG) & 136 & $0,40 \%$ \\
\hline 26 & $\begin{array}{l}\text { Canaris, JZ 2001, } \\
499\end{array}$ & Sondertagung Schuldrechtsmodernisierung & 62 & $0,18 \%$ \\
\hline 98 & $\begin{array}{l}\text { Westermann, NJW } \\
\text { 2002, } 241\end{array}$ & Das neue Kaufrecht & 34 & $0,10 \%$ \\
\hline 125 & $\begin{array}{l}\text { K. Schmidt, NJW } \\
2001,993\end{array}$ & $\begin{array}{l}\text { Die BGB-Außengesellschaft (Bespr. BGH, Az. II ZR } \\
331 / 00 \text { ) }\end{array}$ & 31 & $0,09 \%$ \\
\hline 142 & $\begin{array}{l}\text { Bitter/Meidt, ZIP } \\
\text { 2001, } 2114\end{array}$ & $\begin{array}{l}\text { Nacherfüllungsrecht und Nacherfüllungspflicht des } \\
\text { Verkäufers im neuen Schuldrecht }\end{array}$ & 29 & $0,08 \%$ \\
\hline 155 & $\begin{array}{l}\text { Huber, NJW 2002, } \\
1004\end{array}$ & Der Nacherfüllungsanspruch im neuen Kaufrecht & 28 & $0,08 \%$ \\
\hline
\end{tabular}

Tab. 8: Zitationsrangliste wie Tab. 7, beschränkt auf allgemeine Zeitschriften

Betrachtet man diese Liste näher, fällt zunächst auf, dass deutlich früher als in der Gesamtliste ein Wissenschaftstext auftaucht: Der Aufsatz von C.-W. Canaris ist mit weitem Abstand der meistzitierte Aufsatz, wird er doch in den fünf allgemeinen Zeitschriften von fast doppelt so vielen Artikeln zitiert wie der um 70 Rangplätze abgeschlagene Zweitplatzierte. Auf den ersten Blick verblüfft auch die Tatsache, dass alle fünf meistzitierten Aufsätze aus den Jahren 2001 und 2002 stammen. Das erklärt sich zwanglos daraus, dass vier der fünf Aufsätze die Schuldrechtsmodernisierung (und der fünfte ein lange erwartetes BGH-Urteil) zum Gegenstand haben, und legt Zeugnis ab von der Gesetzgebungs- und Rechtsprechungsnähe der deutschen Jurisprudenz. Damit steht sie in deutlichem Kontrast zur US-amerikanischen Rechtswis- 
senschaft, deren meistzitierte Fachaufsätze zwar sämtlich originelle Grundlagenbeiträge leisten, ${ }^{116}$ dabei aber schon seit Jahrzehnten als esoterisch und praxisfern wahrgenommen werden. ${ }^{117}$

\section{Klassiker der Aufsatzliteratur}

Dass die meistzitierten Aufsätze sehr jung sind und durch ein einziges rechtspolitisches Ereignis dominiert werden, verweist aber auch auf ein methodisches Problem: Weil die hier ausgewerteten Zeitschriften höchstens bis 1981 zurückreichen, werden ältere Texte systematisch benachteiligt. Da juristische Aufsätze in den ersten Jahren nach Erscheinen am häufigsten zitiert werden, ${ }^{118}$ hatten Texte vor 1980 praktisch keine Chance, in die obige Rangliste einzugehen. ${ }^{119}$

Um den Einfluss auch solcher Texte sichtbar zu machen, empfiehlt es sich deshalb, sie nicht mit aktuellen Texten zu vergleichen, sondern mit ihresgleichen. Ein Text darf als umso einflussreicher gelten, je öfter er im Vergleich zu seinen Zeitgenossen zitiert wird. Für diese Auswertung wurde das Erscheinungsjahr in Fünfjahreszeiträume gruppiert und innerhalb jedes dieser Zeiträume der meistzitierte Fachaufsatz in den allgemeinen Zeitschriften ermittelt:

116 Vgl. Shapiro/Pearse, All Time (Fn. 17), S. 1507.

117 H. T. Edwards, The Growing Disjunction Between Legal Education And The Legal Profession, 91 Mich. L. Rev. 1992, S. 34, passim; Rhode, Scholarship (Fn. 92), S. 1342 ff. m.w.N.; jüngst H. G. Manne, How the structure of universities determined the fate of American legal education, Int. Rev. Law Econ. 2014, Suppl. S. 107: "It is almost trite today to catalog the problems of modern legal education [...] inappropriate training for modern practice of law, unneeded and esoteric courses, ideological bias in teaching [usw.]".

118 Für die USA vgl. Zitat oben bei Fn. 58; in den hier ausgewerteten Texten verwiesen gut $41 \%$ aller Zitate auf Fundstellen, die jünger als drei Jahre waren.

119 Vgl. schon Ayres/Vars, Determinants (Fn. 27), S. 447: "A raw citation count has several shortcomings as a measure of an article's impact or quality. The number of citations an article receives is obviously related to how many chances it has had to be cited."; Landes/Posner, Articles (Fn. 26), S. $826 \mathrm{f}$. 


\begin{tabular}{|c|c|c|c|}
\hline Zeitraum & Fundstelle & Titel & Anteil $^{120}$ \\
\hline $\begin{array}{c}1950- \\
1954\end{array}$ & $\begin{array}{l}\text { Doemming/Füßlein/ } \\
\text { Matz, JöR } 1 \text { (1951) } 73\end{array}$ & Entstehungsgeschichte der Artikel des Grundgesetzes & $0,66 \%$ \\
\hline $\begin{array}{c}1955- \\
1959\end{array}$ & $\begin{array}{l}\text { Dürig, AöR } 81 \text { (1956) } \\
117\end{array}$ & Der Grundrechtssatz von der Menschenwürde & $0,62 \%$ \\
\hline $\begin{array}{c}1960- \\
1964\end{array}$ & $\begin{array}{l}\text { Neumann-Duesberg, } \\
\text { JZ 1960, } 114\end{array}$ & $\begin{array}{l}\text { Bildberichterstattung über absolute und relative Per- } \\
\text { sonen der Zeitgeschichte }\end{array}$ & $0,32 \%$ \\
\hline $\begin{array}{c}1965- \\
1969\end{array}$ & Canaris, JZ 1965, 475 & $\begin{array}{l}\text { Ansprüche wegen positiver Vertragsverletzung und } \\
\text { Schutzwirkung für Dritte bei nichtigen Verträgen }\end{array}$ & $0,38 \%$ \\
\hline $\begin{array}{c}1970- \\
1974\end{array}$ & $\begin{array}{l}\text { Böckenförde, NJW } \\
1974,1529\end{array}$ & Grundrechtstheorie und Grundrechtsinterpretation & $0,36 \%$ \\
\hline $\begin{array}{c}1975- \\
1979\end{array}$ & $\begin{array}{l}\text { Stoll, AcP } 176 \text { (1976) } \\
145\end{array}$ & Haftungsverlagerung durch beweisrechtliche Mittel & $0,12 \%$ \\
\hline $\begin{array}{c}1980- \\
1984\end{array}$ & $\begin{array}{l}\text { Canaris, AcP } 184 \\
\text { (1984) } 201\end{array}$ & Grundrechte und Privatrecht & $0,09 \%$ \\
\hline $\begin{array}{c}1985- \\
1989\end{array}$ & $\begin{array}{l}\text { E. Klein, NJW 1989, } \\
1633\end{array}$ & Grundrechtliche Schutzpflicht des Staates & $0,09 \%$ \\
\hline $\begin{array}{c}1990- \\
1994\end{array}$ & Habersack, JuS 1993, 1 & $\begin{array}{l}\text { Die Haftungsverfassung der Gesellschaft bürgerlichen } \\
\text { Rechts - Doppelverpflichtung und Akzessorietät }\end{array}$ & $0,07 \%$ \\
\hline $\begin{array}{c}1995- \\
1999\end{array}$ & $\begin{array}{l}\text { Heinrichs, NJW 1995, } \\
153\end{array}$ & $\begin{array}{l}\text { Umsetzung der EG-Richtlinie über mißbräuchliche } \\
\text { Klauseln in Verbraucherverträgen durch Auslegung }\end{array}$ & $0,06 \%$ \\
\hline $\begin{array}{c}2000- \\
2004\end{array}$ & Canaris, JZ 2001, 499 & $\begin{array}{l}\text { Sondertagung Schuldrechtsmodernisierung. Die Re- } \\
\text { form des Rechts der Leistungsstörungen }\end{array}$ & $0,19 \%$ \\
\hline $\begin{array}{l}2005- \\
2009\end{array}$ & $\begin{array}{l}\text { Lorenz, NJW 2005, } \\
1889\end{array}$ & $\begin{array}{l}\text { Schuldrechtsreform 2002: Problemschwerpunkte drei } \\
\text { Jahre danach }\end{array}$ & $0,08 \%$ \\
\hline
\end{tabular}

Tab. 9: Meistzitierte Wissenschaftstexte in den Fünfjahreszeiträumen seit 1950

Diese Auswertung weist allerdings immer noch Schwächen auf: Zum einen ist die Länge und Abgrenzung von Vergleichszeiträumen rein willkürlich, ${ }^{121}$ zum anderen vernachlässigt sie noch ältere Artikel, aus deren Entstehungszeiträumen generell so wenige Texte zitiert werden, dass sich kaum noch Vergleiche anstellen lassen. Um diese Beschränkungen zu überwinden, bietet sich eine multiplikative Altersgewichtung an. Dazu wird jede Fundstelle genau so oft gezählt, wie Jahre zwischen dem Zeitpunkt ihres Erscheinens und dem ihrer Zitation vergangen sind.

Beispiel: Ein Artikel, der noch sechzig Jahre nach Erscheinen fünf Mal zitiert wird, wird als ebenso einflussreich gewertet wie ein Artikel, der fünf Jahre nach Erscheinen sechzig Mal zitiert wird. Rechnerisch wird also die absolute Häufigkeit, mit der ein Artikel noch x Jahre nach seinem Erscheinen zitiert wurde, mit $x$ multipliziert.

Dieses Vorgehen wertet Fundstellen umso höher, je öfter und je länger sie noch nach ihrem Erscheinen zitiert werden, was zu einer ähnlichen Korrektur wie die oben (bei Fn. 104) durchgeführte Gewichtung von Zeitschriften mit ihrer Materialmenge führt und dadurch die „überragendsten“ Texte der rechtswissenschaftlichen Aufsatzliteratur aufzeigen sollte. Diese Liste beginnt wie folgt:

120 Anteil an allen Zitaten bzgl. Fundstellen aus demselben Fünfjahreszeitraum.

121 Warum Fünfjahreszeiträume seit 1950 vergleichen und nicht Siebenjahreszeiträume seit 1948? 


\begin{tabular}{|c|c|c|}
\hline Rang & Fundstelle & Titel \\
\hline 1 & Radbruch, SJZ 1946, 105 & Gesetzliches Unrecht und übergesetzliches Recht \\
\hline 2 & Heck, AcP 112 (1914) 1 & Gesetzesauslegung und Interessenjurisprudenz \\
\hline 3 & Böckenförde, NJW 1974, 1529 & Grundrechtstheorie und Grundrechtsinterpretation \\
\hline 4 & Canaris, AcP 184 (1984) 201 & Grundrechte und Privatrecht \\
\hline 5 & Dürig, AöR 81 (1956) 117 & Der Grundrechtssatz von der Menschenwürde \\
\hline 6 & Flume, ZHR 136 (1972) 177 & Gesellschaft und Gesamthand \\
\hline 7 & Medicus, JuS 1971, 497 & Durchblick - Die Akzessorietät im Zivilrecht \\
\hline 8 & $\begin{array}{l}\text { Schmidt-Rimpler, AcP } 147 \\
\text { (1941) } 130\end{array}$ & Grundfragen einer Erneuerung des Vertragsrechts \\
\hline 9 & Canaris, JZ 1965, 475 & $\begin{array}{l}\text { Ansprüche wegen positiver Vertragsverletzung und Schutz- } \\
\text { wirkung für Dritte bei nichtigen Verträgen }\end{array}$ \\
\hline 10 & Canaris, JZ 2001, 499 & $\begin{array}{l}\text { Sondertagung Schuldrechtsmodernisierung. Die Reform des } \\
\text { Rechts der Leistungsstörungen }\end{array}$ \\
\hline
\end{tabular}

Tab. 10: Meistzitierte Aufsätze in allgemeinen Zeitschriften, gewichtet mit Alter des Textes bei Zitat

Viele der Texte dürften dem Leser vertraut sein und können mit gutem Recht als Klassiker der Aufsatzliteratur gelten. Drei dieser zehn Klassiker stammen aus der Feder von C.-W. Canaris, und zwar aus drei verschiedenen Jahrzehnten. Nicht zuletzt deshalb darf Canaris als einer der bedeutendsten juristischen Autoren der Gegenwart gelten. ${ }^{122}$

\section{Diskussion}

Zum Abschluss der vorgestellten Pilotstudien sind ihre Grenzen und Verbesserungspotentiale zu thematisieren, bevor zuletzt (E.) die Perspektiven der Zitationsanalyse angedeutet werden.

\section{Geringe Stichprobengröße}

Eine erste Beschränkung der Untersuchung liegt offensichtlich in der geringen Stichprobengröße (so schon oben bei Fn. 113). Obwohl hier über 34.000 Texte ausgewertet wurden, umfasst die Stichprobe für keine Zeitschrift mehr als 33 Jahrgänge (von bis zu 194 möglichen, vgl. oben bei Fn. 88), weshalb Zeitreihenuntersuchungen kaum möglich sind und sämtliche Aussagen zum Zitierverhalten auf die letzten ein bis drei Jahrzehnte beschränkt bleiben müssen. Im Übrigen wurden nur 14 Zeitschriften überhaupt einbezogen, während die konsolidierte Liste der Zeitschriftenkürzel (oben bei Fn. 84) fast hundert Mal so viele Periodika verzeichnet. Zwar ist nicht davon auszugehen, dass sich die oben referierten Ergebnisse qualitativ verschieben würden, wenn mehr Zeitschriften oder weitere Jahrgänge einbezogen würden, doch gesichert ist dies freilich nicht. 


\section{Mangelnde Datenqualität und technische Fehler}

Eine weitere Beschränkung liegt in technischen Mängeln des ausgewerteten Textmaterials. Dazu gehören zunächst Tippfehler im Fußnotentext, die für viele der falschen Fundstellen verantwortlich waren, die in der Datenaufbereitung entfernt werden mussten (oben vor Fn. 88). Der Umfang dieser Tippfehler und das Ausmaß, in dem sie die Auswertung beeinträchtigen, lässt sich praktisch gar nicht abschätzen. Er könnte zwar durch einen Algorithmus ermittelt werden, der jede Fundstelle in einer redaktionellen Fundstellendatenbank nachschlägt, allerdings müssten Datenbankbetreiber dazu entsprechende Schnittstellen anbieten, woran es bislang fehlt.

Desweiteren fehlte bei einigen Zitaten das Erscheinungsjahr, weil es für überflüssig erachtet oder schlicht vergessen wurde. Hier war eine automatisierte Berichtigung praktisch unmöglich, weil bspw. „NJW 2006, 2007“ sowohl die erste Seite des NJWAufsatzes Jahrgang 2006 Seite 2007 bezeichnen könnte als auch (bei fehlender Jahrgangsangabe) die zweite Seite des Aufsatzes Jahrgang 1992 Seite 2006. Umgekehrt wurden auch Texte mehrfach gezählt, die ohne Angabe ihrer Anfangsseite zitiert wurden (z.B. „NJW 1992, 2006“ und „NJW 1992, 2007“ als zwei verschiedene Fundstellen). Während diese Zitierweise bei keiner der ausgewerteten Zeitschriften zur üblichen Redaktionspraxis zu gehören scheint, tauchten doch immer wieder Texte auf, in denen diese Zitierweise mehr oder weniger konsistent eingesetzt wurde. Abhilfe könnte hier wiederum nur eine verlässliche und vollständige Fundstellendatenbank schaffen.

Letztlich erschweren technische Fehler zwar die Datenauswertung und machen oft manuelle Korrekturen oder Sonderalgorithmen erforderlich, allerdings behindern sie die Zitationsanalyse nicht im Ergebnis. Nachdem jeweils von Hand kontrolliert wurde, dass es sich nicht um systematische Abweichungen, sondern nur um zufällig gestreute Fehler handelt, ist davon auszugehen, dass sie sich gegenseitig aufheben und dadurch die Auswertung nicht in einer bestimmten Richtung verzerren.

\section{Beschränkung auf ein einziges Medium}

Eine gravierendere Beschränkung liegt darin, dass die vorliegende Untersuchung nur ein juristisches Publikationsmedium überhaupt erfasst, denn ausgewertet wurden lediglich Zitate aus Zeitschriftenaufsätzen auf Zeitschriften. In beiden Richtungen (Aus welchem Medium heraus wird zitiert? In welches Medium hinein?) findet der juristische Diskurs in vielen weiteren Medienformaten statt, etwa in Kommentaren, Festschriften und anderen Monographien. ${ }^{123}$ Diese können aber mangels einheitlicher Zitierweisen bislang kaum automatisiert ausgewertet werden. Die große Bedeutung von Büchern unterscheidet die juristische Zitationsanalyse maßgeblich von

123 Einschränkend aber Van Gestel/Vranken, Research (Fn. 39), S. 916: "books are still highly important, although perhaps less so than a few decades ago". 
den Naturwissenschaften, ${ }^{124}$ aber auch von der US-amerikanischen Rechtswissenschaft: Dort bieten Zeitschriften nicht selten eine Plattform für „große, eigenständige Entwürfe “, ${ }^{125}$ die hierzulande nur in Monographien ausgearbeitet werden könnten. ${ }^{126}$ Kritiker sehen den Grund für letzteres in den Umfangsbeschränkungen für deutsche Zeitschriftenbeiträge, ${ }^{127}$ deretwegen ihnen „der in Kurzformate gepresste Zeitgeist" innewohne,

„der von den Bundesgerichten mit den zufällig zu ihnen gelangenden Fällen diktiert wird. [...] Die Jurisprudenz wird nicht mehr als Regelungssysteme konstruierende [...] Gedankenkunst begriffen, sondern als Profan-Anleitung für die deutsche Praxis. Eindrucksvolle Beiträge, die man nicht mal eben so überfliegen kann, sondern mit denen man sich beschäftigen muss und über die man spricht, bleiben deshalb weitgehend aus. "128

Will man trotz dieser Kritik nicht leugnen, dass Zeitschriftenbeiträge nennenswert zum Diskurs der deutschen Rechtswissenschaft beitragen, stellt sich immerhin die Frage, welcher Teil des Diskurses unbeobachtet bleibt, wenn ausschließlich Zitate aus Zeitschriften in Zeitschriften betrachtet werden, und wie dieser Dunkelbereich künftig ausgeleuchtet werden könnte.

In der ersten Richtung („Aus welchem Medium heraus wird zitiert?“) lässt sich allenfalls grob abschätzen, welchen Anteil Zeitschriften am Gesamtdiskurs haben: Auf der einen Seite sind gegenwärtig 1.371 Zeitschriften verzeichnet (oben nach Fn. 84), deren Umfang jährlich um je einen oder zwei Bände wachsen dürfte. Dem steht eine jährliche Produktion von etwa 8.000 juristischen Büchern gegenüber. ${ }^{129}$ Damit lässt sich das Verhältnis der Textmengen zueinander natürlich nur grob erahnen, da Satzbild und Seitengrößen enorm variieren - ganz zu schweigen von der Frage, was die jeweils produzierte Textmenge über deren Bedeutung für den Diskurs aussagt. Zumindest für die niederländische Rechtswissenschaft ist allerdings belegt, dass 40 $41 \%$ der juristischen Veröffentlichungen nicht in Zeitschriften erfolgten. ${ }^{130}$ Um deshalb künftig auch Bücher einzubeziehen, müsste deren Text zunächst digital in geeignetem Format vorliegen. Das mag nur noch eine Frage der Zeit sein, allerdings

124 Moed, Citation Analysis (Fn. 8), S. 163: "The role of journals was found to be less prominent in communicating research results in juridical research than it is in many fields in natural and life sciences."; ähnl. schon Landes/Posner, Articles (Fn. 26), S. 826.

125 T. Fuchs, Juristische Gedankenkunst, 4.10.2010, online unter http://blog.delegibus.com/161.

126 Eine gleichwohl extreme Ausnahme ist der berüchtigte Aufsatz von A. S. Jacobs (An Analysis of Section 16 of the Securities Exchange Act of 1934, 32 N. Y. Law School L. Rev. 1987, S. 209-700; 4.824 Fußnoten).

127 Auch US-amerikanische Rechtszeitschriften achten zunehmend auf Kürze: Nachdem in einer Ende 2004 von der Harvard Law Review durchgeführten Befragung die exzessive Länge ihrer Beiträge moniert wurde, kamen Umfangsbeschränkungen (articles length policies) in Mode - meist auf ca. 30.000 Wörter.

128 Fuchs, Gedankenkunst (Fn. 125).

129 Der Katalog der Deutschen Nationalbibliothek verzeichnet 7.721 juristische Bücher für 2011 und 7.451 für 2012 (Suchanfrage $h s g=04 a$ or $h s g=19$ or $h s g=340$, beschränkt auf das jeweilige Jahr und die Materialart „Bücher“, durchgeführt am 16.11.2013).

130 Erhebung 1992-96, zit. in Moed, Citation Analysis (Fn. 8), S. 163. 
lassen die bisherigen Angebote auf dem digitalen Fachbuchmarkt auch eine Entwicklung zu stark nutzungsbeschränkten Textfassungen befürchten, die deren wissenschaftliche Verwendung vielfach beeinträchtigt. ${ }^{131} \mathrm{Ob}$ diese Entwicklung den gleichzeitig zu beobachtenden Drang nach offenen Wissenschaftspublikationen (open access) überlebt, bleibt freilich abzuwarten. Leichter als Monographien könnten Texte der Rechtsprechung einer Zitationsanalyse unterzogen werden, da diese oft schon als digitale Volltexte vorliegen. ${ }^{132}$ Allerdings unterliegen Rechtsprechungstexte keiner redaktionellen Nachkontrolle, weshalb zu befürchten ist, dass ihre Nachweisführung zu willkürlich und nicht systematisch genug erfolgt, um sie sinnvoll quantitativ auszuwerten.

In der zweiten Richtung („In welches Medium hinein wird zitiert?“) lässt sich etwas genauer abschätzen, welcher Teil des Diskurses der Beschränkung auf Zeitschriftenzitate zum Opfer fällt. Geht man davon aus, dass in juristischen Zeitschriften normalerweise mehrere Fundstellen durch Semikola voneinander getrennt werden, lässt sich grob abschätzen, wieviele separate Fundstellen in der vorliegenden Stichprobe insgesamt zitiert wurden, indem zur Anzahl der Fußnoten (1.671.243, oben bei Fn. 77) die Anzahl aller verwendeten Semikola (1.201.683) addiert wird. Vergleicht man die Summe von etwa 2,87 Millionen Fundstellen mit den hier als Zeitschriftenzitat identifizierten 1,62 Millionen Fundstellen (oben nach Fn. 86), so ergibt sich ein Verhältnis von 56 \%. Diese krude Schätzung legt nahe, dass die vorliegende Untersuchung zumindest innerhalb der untersuchten Zeitschriften etwa jede zweite Fundstelle erfasst hat. Um auch die übrigen auszuwerten, müssten sich Algorithmen zur Herauslösung von Zitaten finden lassen, die auf andere Publikationen als Zeitschriften verweisen. Das scheitert bislang vor allem daran, dass es für diese Publikationen keine ähnlich etablierten Zitierformate wie für Zeitschriften gibt. Mit deren Entwicklung ist wohl auch kaum noch zu rechnen. Entscheidende Fortschritte in dieser Richtung könnte lediglich die zunehmende Vernetzung und Verlinkung digitaler Inhalte bringen, die eine manuelle Kodierung von Fundstellen in einer maschinenlesbaren Form erfordert. Das erfolgt vor allem im „unsichtbaren“ Metatext der Dokumente und könnte von dort unmittelbar in entsprechende Algorithmen übernommen werden. Eine solche redaktionelle Aufbereitung würde auch viele weitere Beschränkungen der vorliegenden Datengrundlage beseitigen und ganz neue Auswertungsmöglichkeiten eröffnen.

\section{E. Ausblick: Die Zukunft der Zitationsanalyse}

Die vorliegenden Studien sind ein Pilotprojekt. Sie sollen die gezielte Diskussion über andere Wege der juristischen Forschungsevaluation ${ }^{133}$ weder erübrigen noch vor-

131 Aus diesem Grund wurden vorliegend bspw. keine Inhalte der Jurion-Datenbank ausgewertet.

132 Vgl. die frühen Studien in Fn. 28 sowie Siems, Wie häufig (Fn. 7), S. 91 bei Fn. 8 m.w.N. in Fn. 3.

133 Dazu ausf. mit zahlreichen Anregungen Van Gestel/Vranken, Research (Fn. 39). 
wegnehmen, sondern gerade dazu beitragen. Das weitere Schicksal der juristischen Zitationsanalyse entscheidet sich aber nicht nur an ihrer wahrgenommenen Nützlichkeit, sondern mehr noch an der Verfügbarkeit und Qualität der dafür zur Verfügung stehenden Daten. ${ }^{134}$

Für die vorliegenden Studien umfasste der Entwicklungsprozess von der Datenbankabfrage bis zur fertigen Auswertung ein Dutzend sehr aufwändige Arbeitsschritte, für die komplett neue Algorithmen entworfen und durch permanentes Experimentieren verbessert werden mussten, denn auf Vorlagen oder Schnittstellen konnte an keiner Stelle zurückgegriffen werden (vgl. oben Fn. 83, 86). Mit jeder Änderung der Algorithmen musste die Auswertungsroutine ganz oder in großen Teilen neu durchlaufen werden; allein dieser Schritt dauerte auf einem handelsüblichen PC mehrere Stunden. Mit einer dreistelligen Anzahl Arbeitsstunden lag der Aufwand für die vorliegende Studie deshalb in der Nähe dessen, was ein Einzelner überhaupt bewältigen mag. Ähnlicher Aufwand wäre zu erwarten, wenn andere Zeitschriften oder weitere Medienformate in die Auswertung einbezogen würden. Dieser Aufwand war allenfalls als Pionierarbeit zu rechtfertigen und steht für den bisherigen Ertrag noch erkennbar außer Verhältnis.

Das bedeutet allerdings nicht, dass die Zitationsanalyse schon an ihrer mangelnden Durchführbarkeit scheitern müsste. Die neuere juristische Zitationsanalyse in den USA begegnete anfangs ähnlichen Herausforderungen: Als deren Konsumenten andere Datenquellen und anspruchsvollere Auswertungsmethoden nachfragten, erwies sich das zunächst als undurchführbar:

"My response had always been to acknowledge that lists of most-cited scholars and books would be more interesting than ones of articles, but to point out that compiling such lists would be probibitively difficult." 135

Das änderte sich erst, als ein Fachverlag „mit ungewöhnlicher Großzügigkeit“ ermöglichte, „eine mächtige Datenbank juristischer Zitationsinformationen zu nutzen “. ${ }^{136}$ Denn verlagsseitig liegen viele der für eine Zitationsanalyse erforderlichen Informationen bereits redaktionell aufbereitet vor, und könnten für die Wissenschaft von großem Nutzen sein. Eine solche Aufbereitung dürfte viele der weiteren oben genannten Beschränkungen beseitigen und ganz neue Fragestellungen ermöglichen. ${ }^{137}$ Denn im Prinzip sind die juristischen Arbeitsmittel sogar

$134 \mathrm{Zu}$ diesem Hindernis schon Hanau/Gotthardt, Evaluation (Fn. 42), S. 173.

135 Shapiro, Papers (Fn. 15), S. vi.

136 Shapiro, Papers (Fn. 15), S. vi (Übersetzung H.H.).

137 Über die Zitationsanalyse hinaus käme eine solche Aufbereitung auch allgemein der Korpuslinguistik zugute, also der auf große Textsammlungen angewandten Computerlinguistik - wie der von der Heidelberger Akademie der Wissenschaften geförderten Konzeption und Erschließung eines juristischen Referenzkorpus, vgl. http://www.jureko.de. 
"superior to those in other disciplines, such as extensive full-text online databases which have highly flexible capabilities for retrieving references to any source or combination of sources and references in conjunction with desired words. Therefore in law, the birthplace of citation study, even richer results may be possible than in the other fields to which that study has subsequently been applied."138

Mit verlagsseitiger Unterstützung und sorgfältig koordinierten Forschungsprojekten könnten zitationsanalytische Instrumente schon bald zum Werkzeugkoffer der juristischen Textarbeit gehören.

138 Shapiro, Origins (Fn. 9), S. 339. 